\title{
Commuting difference operators with elliptic coefficients from Baxter's vacuum vectors
}

\author{
A. Zabrodin *
}

December 1999

\begin{abstract}
For quantum integrable models with elliptic $R$-matrix, we construct the Baxter $Q$-operator in infinite-dimensional representations of the algebra of observables.
\end{abstract}

\section{Introduction}

The method of $Q$-operator, first proposed by Baxter in his solution of the 8-vertex model and the spin- $\frac{1}{2} X Y Z$ chain [1], is perhaps the most powerful approach to quantum integrable models known today. As compared with the algebraic Bethe ansatz [2], one or another version of this approach, known also as the method of vacuum vectors, is indispensable for models which do not possess bare vacuum states. Among later striking developments we mention the works [3], where the Baxter method was applied to the chiral Potts model.

At that time, however, the $Q$-operator itself seemed to be a kind of virtual object for its explicit form was not known. In Baxter's original work, the $Q$-operator played an auxiliary role since the local Hamiltonian to be diagonalized was made of the quantum transfer matrix $\mathbf{T}(\lambda)$. The main properties of the $Q$-operator $\mathbf{Q}(\lambda)$ are the commutativity $[\mathbf{Q}(\lambda), \mathbf{Q}(\mu)]=0,[\mathbf{Q}(\lambda), \mathbf{T}(\mu)]=0$ for all $\lambda, \mu$, and the following $T$ - $Q$-relation:

$$
\mathbf{T}(\lambda) \mathbf{Q}(\lambda)=a(\lambda) \mathbf{Q}(\lambda+\eta)+b(\lambda) \mathbf{Q}(\lambda-\eta) .
$$

Here $a(\lambda), b(\lambda)$ are $c$-number functions depending on a particular model. Combined with (model-dependent) analytical properties of the $\mathbf{Q}(\lambda)$, this relation allows one to obtain a system of Bethe equations and eventually to solve the diagonalization problem. In the absense of global bare vacuum states, Baxter's method to derive (1.1) was to consider a continuous-parametric family of special states that factorize into products of local vacuum vectors. Such states have especially simple properties under action of $\mathbf{T}(\lambda)$. Treating them as columns or rows of some matrix $\mathbf{Q}(\lambda)$, one arrives, after a proper normalization, at (1.1). Some years ago this approach was extended [4] to the case of twisted boundary

* Joint Institute of Chemical Physics, Kosygina str. 4, 117334, Moscow, Russia and ITEP, 117259, Moscow, Russia 
conditions. It remained obscure, however, whether the $Q$-matrix could play any other role than just a technical tool for deriving Bethe equations.

The situation changed only quite recently, when it was realized that in integrable lattice models with an infinite-dimensional Hilbert space at each site it is $\mathbf{Q}$ rather than $\mathbf{T}$ that is the generating function of local Hamiltonians. With this motivation in mind, a considerable progress in constructing the $Q$-operator for a number of integrable models and in understanding its nature was achieved.

The work [5] was perhaps the first, where the Baxter approach was adopted to integrable models with an infinite-dimensional quantum space. Specifically, in that paper the $Q$-operator for the quantum Toda chain was constructed as certain integral operator with explicitly known kernel. Recently, this remarkable development was extended [6] to the integrable homogeneous $X X X$ spin chain in a unitary discrete series representation of the non-compact group $S U(1,1)$ (see also [7]).

For integrable models of $X X Z$-type, the nature of $Q$-operator was clarified in [8], [9], where it was given a representation theory meaning. Specifically, the $Q$-operator can be obtained from the universal $R$-matrix for the quantum affine algebra $U_{q}(\widehat{s l(2)})$, by taking trace of a chain product of the universal $R$-matrices in a special $q$-oscillator representation in one of the spaces. The commutativity of the $Q$-operators, which comes out as a surprize in Baxter's approach, is now guaranteed by characteristic properties of the universal $R$-matrix. The $T-Q$ relation, from this point of view, is a simple consequence of fusion rules for representations of the quantum affine algebra. In fact, two different commuting $Q$-operators, $\mathbf{Q}^{+}(\lambda)$ and $\mathbf{Q}^{-}(\lambda)$, can be introduced this way. They are two linearly independent operator solutions to the $T$ - $Q$-relation (1.1) treated as a second order difference equation in $\lambda$. As such, they obey a quantum Wronskian condition, which turns out to be even more instructive than the $T$ - $Q$ relation itself.

As for matters more customary for theory of solitons, it was argued in [10, 11] that $Q$-operator is a quantum analog of Bäcklund transformation. In other words, it defines an integrable quantum model with discrete time by setting $\mathbf{Q}(\lambda)$ to be the time shift operator. For any observable $\mathbf{O}$, one sets $\mathbf{O}(t+\delta)=\mathbf{Q}(\lambda) \mathbf{O}(t)(\mathbf{Q}(\lambda))^{-1}$, where the discrete time shift $\delta$ is a function of $\lambda$. Integrability implies that these discrete evolution operators commute for all $\delta$.

However, all this was about models associated with rational or trigonometric solutions of the Yang-Baxter equation ( $R$-matrices). General $R$-matrices with additive spectral parameter are parametrized via elliptic functions. The symplest elliptic $R$-matrix is

$$
\mathrm{R}(\lambda)=\sum_{a=0}^{3} \frac{\theta_{a+1}(2 \lambda+\eta \mid \tau)}{\theta_{a+1}(\eta \mid \tau)} \sigma_{a} \otimes \sigma_{a},
$$

where $\theta_{a}(z \mid \tau)$ are Jacobi $\theta$-functions, $\sigma_{a}$ are Pauli matrices, and $\sigma_{0}$ is the unit matrix. This $R$-matrix is associated with the 8 -vertex model, being the matrix of local Boltzmann weights at a vertex. The transfer matrix of this model is the generating function of conserved quantities for the integrable $X Y Z$ spin- $\frac{1}{2}$ chain. By models of the $X Y Z$-type we understand more general integrable models with elliptic $R$-matrix, for instance integrable anisotropic spin chains with higher spin. Integrable spin chains of $X Y Z$-type can be solved by the generalized algebraic Bethe ansatz [2], [12].

However, the quantum affine algebra approach is not applicable in the $X Y Z$-case since 
nothing similar to the universal elliptic $R$-matrix is known. Nevertheless, the elliptic dependence on the spectral parameter does not destroy the functional relations between transfer matrices and $Q$-operators, including the $T$ - $Q$ relation and the Wronskian condition [13], [14]. This suggests that everything what is possible to do in $X X X$ and $X X Z$ cases, might be possible, mutatis mutandis, for $X Y Z$, too.

In this paper we present a direct construction of the $Q$-operator for homogeneous $N$ site spin chains of the $X Y Z$-type. In lattice integrable models with elliptic $R$-matrix, the algebra of local observables is the Sklyanin algebra [15, 16]. A concrete model is defined by fixing a particular representation of this algebra. Similar to the $s l(2)$-case (models of $X X X$-type), the representations are labeled by a continuous parameter which is called spin, by analogy with $\operatorname{sl}(2)$. We allow the spin to take any complex value, so we work in an infinite-dimensional representation of the algebra of observables. In other words, we deal with representations of what would be called a non-compact real form of the Sklyanin algebra. Such representations can be realized by difference operators, so our $Q$-operator is a diffeference operator in many variables.

Our strategy closely follows the original Baxter argument. First, we construct a family of local (infinite-dimensional) vacuum vectors that obey the celebrated "pair propagation through a vertex" property. Next, their product over all sites of the chain is, up to normalization, the kernel of the $Q$-operator. At last, one should prove the commutativity of the $Q$-operators. However, the practical realization of these ideas in the infinite-dimensional setting is by no means obvious. Technically, it is very different from what is customary in the 8-vertex model. The construction extensively uses such truly special functions as elliptic generalizations of the gamma-function and basic hypergeometric functions, and highly non-trivial identities for them. For example, commutativity of the $Q$-operators relies on an elliptic version of the Bailey transformation formula for the very-well-poised basic hypergeometric series ${ }_{10} W_{9}$. The appearence of such a sophisticated mathematics in integrable models is not so surprizing. Recently, the elliptic series ${ }_{10} W_{9}$ was shown [17] to offer a natural representation for "elliptic 6 - $j$-symbols", which are nothing else than solutions of the Yang-Baxter equation in a special basis.

It is now a challenge to reveal a representation theory meaning of the elliptic $Q$ operator. Hopefully, this would make its properties and their proofs obvious.

The paper is organized as follows. In the next section we present the main result in the shortest possible form. Sec. 3 contains the necessary material on rerpesentations of the Sklyanin algebra. The construction of the $Q$-operator begins in Sec. 4 , where we find a family of local vacuum states for the quantum L-operator. In Sec. 5 we investigate direct products of such states along the chain and define unnormalized "pre- $Q$-operators", $\mathbf{Q}_{L}$ and $\mathbf{Q}_{R}$, which do not yet commute. Sec. 6 is rather technical. It is devoted to the proof of the basic commutation relation for the pre- $Q$-operators, $\mathbf{Q}_{L}(\lambda) \mathbf{Q}_{R}(\mu)=\mathbf{Q}_{L}(\mu) \mathbf{Q}_{R}(\lambda)$, which entails commutativity of the $Q$-operators introduced in Sec.7. In Sec. 8 we discuss more specific issues: a) twisted boundary conditions, b) the exceptional case of a "spin chain" with only one site, $N=1$, and c) the trigonometric ( $X X Z$-type) degeneration. A few concluding remarks are given in Sec. 9.

Appendix A contains necessary information on the special functions involved in the construction. In Appendix B we give a direct proof of the intertwining relation between representations of spins $\ell$ and $-\ell-1$. 


\section{The main result}

To state the result, we recall the definition of the quantum transfer matrix $\mathbf{T}(\lambda)$ for the $X Y Z$ spin chain with spin $\ell$. The elliptic quantum L-operator is the matrix

$$
\mathbf{L}(\lambda)=\frac{1}{2}\left(\begin{array}{cc}
\theta_{1}(2 \lambda) \mathbf{s}_{0}+\theta_{4}(2 \lambda) \mathbf{s}_{3} & \theta_{2}(2 \lambda) \mathbf{s}_{1}+\theta_{3}(2 \lambda) \mathbf{s}_{2} \\
\theta_{2}(2 \lambda) \mathbf{s}_{1}-\theta_{3}(2 \lambda) \mathbf{s}_{2} & \theta_{1}(2 \lambda) \mathbf{s}_{0}-\theta_{4}(2 \lambda) \mathbf{s}_{3}
\end{array}\right)
$$

with non-commutative matrix elements. Specifically, $\mathbf{s}_{a}$ are difference operators in a complex variable $z$ :

$$
\mathbf{s}_{a}=\frac{\theta_{a+1}(2 z-2 \ell \eta)}{\theta_{1}(2 z)} e^{\eta \partial_{z}}-\frac{\theta_{a+1}(-2 z-2 \ell \eta)}{\theta_{1}(2 z)} e^{-\eta \partial_{z}}
$$

introduced by Sklyanin [16]. Here $\theta_{a}(z) \equiv \theta_{a}(z \mid \tau)$ are Jacobi $\theta$-functions with the elliptic module $\tau, \operatorname{Im} \tau>0, \ell$ is a complex number (the spin), and $\eta \in \mathbb{C}$ is a parameter which is assumed to belong to the fundamental parallelogram with vertices $0,1, \tau, 1+\tau$, and to be incommensurate with $1, \tau$. Definitions and transformation properties of the $\theta$-functions are listed in Appendix A.

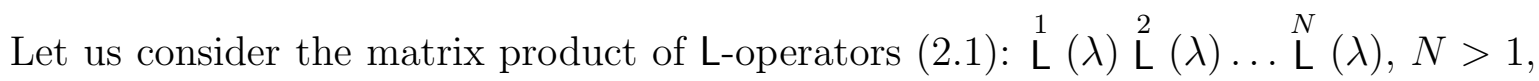
where each operator matrix $L^{i}(\lambda)$ has the form (2.1) with $\mathbf{s}_{a}$ acting on its own variable $z_{i}$. Clearly, matrix elements of $L^{i}(\lambda)$ and ${ }^{j}(\lambda)$ commute for $i \neq j$. The trace of this product (the sum of two diagonal elements),

$$
\mathbf{T}(\lambda)=\operatorname{tr}\left[\stackrel{1}{\mathrm{~L}}(\lambda) \stackrel{2}{\mathrm{~L}}(\lambda) \ldots \stackrel{N}{\mathrm{~L}}^{\prime}(\lambda)\right]
$$

is called the quantum transfer matrix of the spin chain.

Our main result is the explicit realization of the $Q$-operator for this spin chain:

$$
\mathbf{Q}(\lambda)=\Gamma^{-N}(2 \lambda-2 \ell \eta): \prod_{i=1}^{N} \mathbf{q}_{i, i+1}(\lambda): e^{(\lambda+\ell \eta) \sum_{j=1}^{N} \partial_{z_{j}}}
$$

where

$$
\begin{aligned}
\mathbf{q}_{i, i+1}(\lambda) & =\frac{\Gamma\left(2 z_{i}\right) \Gamma\left(z_{i}-z_{i+1}+2 \lambda\right) \Gamma\left(z_{i}+z_{i+1}+2 \lambda\right)}{\Gamma\left(2 z_{i}+2 \lambda+2 \ell \eta\right) \Gamma\left(z_{i}-z_{i+1}-2 \ell \eta\right) \Gamma\left(z_{i}+z_{i+1}-2 \ell \eta\right)} \times \\
& \times{ }_{6} W_{5}\left(-\frac{z_{i}+\lambda}{\eta}-\ell ;-\frac{\lambda}{\eta}-\ell,-\frac{z_{i+1}+z_{i}}{2 \eta}-\ell, \frac{z_{i+1}-z_{i}}{2 \eta}-\ell ; e^{-2 \eta z_{z_{i}}} \mid \eta, \tau\right)
\end{aligned}
$$

(it is implied that $\left.z_{N+1}=z_{1}\right)$. Here $\Gamma(z)=\Gamma(z \mid \tau, 2 \eta)$ is the elliptic gamma-function [18, 19":

$$
\Gamma(z \mid \tau, 2 \eta)=\prod_{k, m=0}^{\infty} \frac{1-e^{2 \pi i((k+1) \tau+2(m+1) \eta-z)}}{1-e^{2 \pi i(k \tau+2 m \eta+z)}}=\exp \left(-i \sum_{k=1}^{\infty} \frac{\sin (\pi k(2 z-2 \eta-\tau))}{2 k \sin (2 \pi k \eta) \sin (\pi k \tau)}\right),
$$


and ${ }_{6} W_{5}\left(\alpha_{1} ; \alpha_{4}, \ldots, \alpha_{6} ; z \mid \eta, \tau\right)$ is the elliptic analog of the very-well-poised hypergeometric series (with the operator argument):

$$
{ }_{6} W_{5}\left(\alpha_{1} ; \alpha_{4}, \ldots, \alpha_{6} ; z \mid \eta, \tau\right)=\sum_{k=0}^{\infty} \frac{\left[\alpha_{1}+2 k\right]\left[\alpha_{1}\right]_{k}}{\left[\alpha_{1}\right][1]_{k}}\left(\prod_{m=1}^{3} \frac{\left[\alpha_{m+3}\right]_{k}}{\left[\alpha_{1}-\alpha_{m+3}+1\right]_{k}}\right) z^{k}
$$

where $[\alpha]_{k}=\prod_{j=0}^{k-1} \theta_{1}(2 \alpha \eta+2 j \eta)$. For more information on the special functions $\Gamma,{ }_{6} W_{5}$ see Appendix A. The normal ordering means that all operators $e^{-2 \eta \partial_{z_{j}}}$ are moved to the right.

We prove the commutativity $[\mathbf{Q}(\lambda), \mathbf{T}(\mu)]=[\mathbf{Q}(\lambda), \mathbf{Q}(\mu)]=0$, and the $T-Q$ relation

$$
\mathbf{T}(\lambda) \mathbf{Q}(\lambda)=\theta_{1}^{N}(2 \lambda-2 \ell \eta) \mathbf{Q}(\lambda+\eta)+\theta_{1}^{N}(2 \lambda+2 \ell \eta) \mathbf{Q}(\lambda-\eta)
$$

Another solution to this operator difference equation has a similar form (see (7.8)).

The limiting case $\tau \rightarrow 0$, when elliptic functions degenerate to trigonometric ones, corresponds to the $X X Z$ spin chain. The structure of the formulas (2.4), (2.5) remains the same while the special functions get simplified. The elliptic gamma-function becomes the $q$-gamma function (or the $q$-exponential function) while the elliptic hypergeometric series ${ }_{6} W_{5}$ turns into the little $q$-Jacobi function represented by the basic hypergeometric series ${ }_{2} \phi_{1}$.

\section{Representations of the Sklyanin algebra}

The aim of this section is to give the necessary preliminaries on representations of the Sklyanin algebra. We begin with a few formulas related to the quantum L-operator with elliptic dependence on the spectral parameter.

Consider the quantum L-operator (2.1), where $\mathbf{s}_{a}$ are realized as in (2.2). The four operators $\mathbf{s}_{a}$ obey the commutation relations of the Sklyanin algebra円:

$$
\begin{aligned}
& (-1)^{\alpha+1} I_{\alpha 0} \mathbf{s}_{\alpha} \mathbf{s}_{0}=I_{\beta \gamma} \mathbf{s}_{\beta} \mathbf{s}_{\gamma}-I_{\gamma \beta} \mathbf{s}_{\gamma} \mathbf{s}_{\beta}, \\
& (-1)^{\alpha+1} I_{\alpha 0} \mathbf{s}_{0} \mathbf{s}_{\alpha}=I_{\gamma \beta} \mathbf{s}_{\beta} \mathbf{s}_{\gamma}-I_{\beta \gamma} \mathbf{s}_{\gamma} \mathbf{s}_{\beta}
\end{aligned}
$$

with the structure constants $I_{a b}=\theta_{a+1}(0) \theta_{b+1}(2 \eta)$. Here $a, b=0, \ldots, 3$ and $\{\alpha, \beta, \gamma\}$ stands for any cyclic permutation of $\{1,2,3\}$. The relations of the Sklyanin algebra are equivalent to the condition that the L-operator satisfies the "RLL $=$ LLR" relation with the elliptic $R$-matrix (1.2).

Plugging the difference operators (2.2) into (2.1), one can represent the L-operator in a factorized form, which is especially convenient for computations:

$$
\mathrm{L}(\lambda)=\theta_{1}\left(2 \lambda_{+}\right): V^{-1}\left(\lambda_{+}, z\right)\left(\begin{array}{cc}
e^{\eta \partial_{z}} & 0 \\
0 & e^{-\eta \partial_{z}}
\end{array}\right) V\left(\lambda_{-}, z\right):
$$

\footnotetext{
${ }^{1}$ The standard generators of the Sklyanin algebra [15, $S_{a}$, are related to ours as follows: $S_{a}=$ $(i)^{\delta_{a, 2}} \theta_{a+1}(\eta) \mathbf{s}_{a}$.
} 
Hereafter we use the convenient notation $\lambda_{ \pm} \equiv \lambda \pm \ell \eta, \bar{\theta}_{a}(z) \equiv \theta_{a}\left(z \mid \frac{\tau}{2}\right)$. The dots mean the normal ordering when the shift operators are moved to the right and

$$
V(\lambda, z)=\left(\begin{array}{cc}
\bar{\theta}_{4}(z+\lambda) & \bar{\theta}_{3}(z+\lambda) \\
\bar{\theta}_{4}(z-\lambda) & \bar{\theta}_{3}(z-\lambda)
\end{array}\right)
$$

Note that

$$
\mathrm{L}\left(-\lambda+\frac{1}{2}(1+\tau)\right)=-e^{-\pi i \tau+4 \pi i \lambda} \mathrm{L}^{\top}(\lambda)
$$

where $\mathrm{L}^{\top}$ means transposition in the two-dimensional space.

The parameter $\ell$ in (2.2) is called the spin of the representation. If necessary, we write $\mathbf{s}_{a}=\mathbf{s}_{a}^{(\ell)}$ to indicate the dependence on $\ell$. When $\ell \in \frac{1}{2} \mathbb{Z}_{+}$, these operators have a finite-dimensional invariant subspace, namely, the space $\Theta_{4 \ell}^{+}$of even $\theta$-functions of order $4 \ell$ (see Appendix A). This is the representation space of the $(2 \ell+1)$-dimensional irreducible representation (of series a)) of the Sklyanin algebra. For example, at $\ell=\frac{1}{2}$ the functions $\bar{\theta}_{4}(z), \bar{\theta}_{3}(z)$ form a basis in $\Theta_{2}^{+}$, and the generators $\mathbf{s}_{a}$, with respect to this basis, are represented by $2 \times 2$ matrices $(-i)^{\delta_{a, 2}}\left(\theta_{a+1}(\eta)\right)^{-1} \sigma_{a}$. In this case, $\mathrm{L}(\lambda)=\mathrm{R}\left(\lambda-\frac{1}{2} \eta\right)$, where $\mathrm{R}$ is the 8 -vertex model $R$-matrix (1.2).

As is proved in [20], the space $\Theta_{4 \ell}^{+}$for $\ell \in \frac{1}{2} \mathbb{Z}_{+}$is annihilated by the operator

$$
\mathbf{w}_{\ell}=\sum_{k=0}^{2 \ell+1}(-1)^{k}\left[\begin{array}{c}
2 \ell+1 \\
k
\end{array}\right] \frac{\theta_{1}(2 z+2(2 \ell-2 k+1) \eta)}{\prod_{j=0}^{2 \ell+1} \theta_{1}(2 z+2(j-k) \eta)} e^{(2 \ell-2 k+1) \eta \partial_{z}}
$$

Here and below we use the "elliptic factorial" and "elliptic binomial" notation:

$$
[j] \equiv \theta_{1}(2 j \eta), \quad[n] !=\prod_{j=1}^{n}[j], \quad\left[\begin{array}{c}
n \\
m
\end{array}\right] \equiv \frac{[n] !}{[m] ![n-m] !} .
$$

Another important property of the operator $\mathbf{w}_{\ell}$ established in [20] is that $\mathbf{w}_{\ell}$ intertwines representations of spin $\ell$ and of spin $-(\ell+1)$ :

$$
\mathbf{s}_{a}^{(-\ell-1)} \mathbf{w}_{\ell}=\mathbf{w}_{\ell} \mathbf{s}_{a}^{(\ell)}, \quad a=0, \ldots, 3 .
$$

The same intertwining relation can be written for the quantum L-operator (2.1). The operator $\mathbf{w}_{\ell}$ is an elliptic analog of $(d / d z)^{2 \ell+1}$ in the following sense. In the case of the algebra $s l_{2}$, the intertwining operator between representations of spins $\ell$ and $-\ell-1$ (realized by differential operators in $z$ ) is $(d / d z)^{2 \ell+1}$. It annihilates the linear space of polynomials of degree $\leq 2 \ell$.

The operator $\mathbf{w}_{\ell}$ can be extended to arbitrary complex values of $\ell$ in which case it is represented by a half-infinite series in the shift operator $e^{2 \eta \partial_{z}}$. The series is an elliptic analog of the very-well-poised basic hypergeometric series with an operator argument. For its explicit form see (7.13). The intertwining relations (3.6) hold true in this more general case, too. The proof is outlined in Appendix B.

In what follows we need infinite dimensional representations of the Sklyanin algebra in some functional space. Operators (2.2) provide such a representation. However, they are not sufficient for our purpose since it is necessary to specify the functional space where these operators are going to act. It is not clear how to do that for continuous functions. On the other hand, the difference character of the operators (2.2) allows one to restrict them to discontinuous functions of the form described below. 
Let $\delta(z)$ be the function equal to zero everywhere but at $z=0$, where it equals 1 : $\delta(z)=0, z \neq 0, \delta(0)=1$. Clearly, $z \delta(z)=0$. The Sklyanin algebra realized as in (2.2) acts, for any $\nu \in \mathbb{C}$, in the space of functions of the form

$$
f(z)=\sum_{k \in \mathbb{Z}} f_{k} \delta(z-\nu+2 k \eta), \quad f_{k} \in \mathbb{C} .
$$

This space is isomorphic to the linear space of sequences $\left\{f_{k}\right\}_{k \in \mathbb{Z}}$. We call functions of the form (3.7) combs.

A comb is said to be finite from the right (respectively, from the left) if there exists $M \in \mathbb{Z}$ such that $f_{k}=0$ as $k>M$ (respectively, $k<M$ ). Let $\mathcal{C}^{\vdash}$ (respectively, $\mathcal{C}^{\dashv}$ ) be the space of combs finite from the left (respectively, from the right).

We define the pairing

$$
(F(z), \delta(z-a))=F(a)
$$

for any function $F(z)$, not necessarily of the form (3.7). In particular,

$$
(\delta(z-a), \delta(z-b))=\delta(a-b) .
$$

Formally, this pairing can be written as an integral:

$$
(F(z), \delta(z-a))=\int F(z) \delta(z-a) d z
$$

(perhaps a $q$-integral symbol would be more appropriate). We stress that the integral here means nothing more than another notation for the pairing, especially convenient in case of many variables. By linearity, the pairing can be extended to the whole space of combs. We note that the pairing between the spaces $\mathcal{C}^{\vdash}$ and $\mathcal{C}^{\dashv}$ is well defined since the sum is always finite.

Combs are to be viewed as kernels of difference operators. By a difference operator in one variable we mean any expression of the form

$$
\mathbf{D}=\sum_{k \in \mathbb{Z}} c_{k}(z) e^{(\mu+2 k \eta) \partial_{z}}, \quad \mu \in \mathbb{C} .
$$

The comb

$$
D(z, \zeta)=\sum_{k \in \mathbb{Z}} c_{k}(z) \delta(z-\zeta+\mu+2 k \eta),
$$

regarded as a function of any one of the variables $z, \zeta$, is the kernel of this difference operator in the following sense. Using the pairing introduced above, we can write:

$$
(\mathbf{D} f)(z)=\int D(z, \zeta) f(\zeta) d \zeta=\sum_{k \in \mathbb{Z}} c_{k}(z) f(z+\mu+2 k \eta) .
$$

The kernel $D(z, \zeta)$ can be viewed as an infinite matrix with continuously numbered rows $(z)$ and columns $(\zeta)$. Then the convolution with respect to the second argument of the kernel, as in (3.12), defines action of the operator from the left. The convolution with respect to the first argument defines the action from the right,

$$
(f \mathbf{D})(z)=\int f(\zeta) D(\zeta, z) d \zeta,
$$


equivalent to the action of the transposed difference operator from the left:

$$
\mathbf{D}^{\mathbf{t}}=\sum_{k \in \mathbb{Z}} e^{-(\mu+2 k \eta) \partial_{z}} c_{k}(z)=\sum_{k \in \mathbb{Z}} c_{k}(z-\mu-2 k \eta) e^{-(\mu+2 k \eta) \partial_{z}}
$$

The transposition $\mathrm{t}$ is the anti-automorphism of the algebra of difference operators such that $\left(c(z) e^{\alpha \partial_{z}}\right)^{\mathrm{t}}=e^{-\alpha \partial_{z}} c(z)$. In terms of the above pairing we can write $(f, \mathbf{D} g)=\left(\mathbf{D}^{\mathrm{t}} f, g\right)$.

The kernels of Sklyanin's operators (2.2) are:

$$
s_{a}\left(z, z^{\prime}\right)=\frac{\theta_{a+1}(2 z-2 \ell \eta)}{\theta_{1}(2 z)} \delta\left(z-z^{\prime}+\eta\right)-\frac{\theta_{a+1}(-2 z-2 \ell \eta)}{\theta_{1}(2 z)} \delta\left(z-z^{\prime}-\eta\right) .
$$

Let us also note that $\mathbf{s}_{a}^{(\ell) \mathbf{t}}=-(-1)^{\delta_{a, 0}} \theta_{1}(2 z) \mathbf{s}_{a}^{(-\ell-1)}\left(\theta_{1}(2 z)\right)^{-1}$.

\section{Local vacuum vectors}

We are going to adopt the Baxter method of vacuum vectors to the infinite-dimensional representations of the Sklyanin algebra described in the previous section.

Let us recall the general definition of vacuum vectors. Consider an arbitrary L-operator $\mathrm{L}$ with two-dimensional auxiliary space $\mathbb{C}^{2}$, i.e., an arbitrary $2 \times 2$ operator-valued matrix whose matrix elements are operators $\mathbf{L}_{11}, \mathbf{L}_{12}, \mathbf{L}_{21}, \mathbf{L}_{22}$ :

$$
\mathbf{L}=\left(\begin{array}{ll}
\mathbf{L}_{11} & \mathbf{L}_{12} \\
\mathbf{L}_{21} & \mathbf{L}_{22}
\end{array}\right)
$$

They act in a linear space $\mathcal{H}$ which is called the quantum space of the L-operator.

For a moment, let $\psi, \phi$ denote vectors from $\mathbb{C}^{2}$. Let $X \in \mathcal{H}$, then acting by the quantum L-operator on the tensor product $X \otimes \phi$, we, generally speaking, obtain a mixed state in the quantum space: $\mathrm{L} X \otimes \phi=X_{1} \otimes \phi_{1}+X_{2} \otimes \phi_{2}$. The special case of a pure state,

$$
\mathrm{L} X \otimes \phi=X^{\prime} \otimes \psi
$$

is of particular importance. Given an L-operator, Baxter's receipe is to find all pairs $X, \phi$ such that (4.2) holds. Taking the scalar product with the vector $\psi^{\perp}$ orthogonal to $\psi$, we get:

$$
\left(\psi^{\perp} \mathrm{L} \phi\right) X=0
$$

i.e., the operator $\mathbf{K}=\left(\psi^{\perp} \mathrm{L} \phi\right)$ (acting in the quantum space only) has a zero mode $X \in \mathcal{H}$. Suppose (4.2) (or (4.3)) holds with some vectors $\phi, \psi$; then the vector $X$ is called a vacuum vector of the $L$-operator.

A few remarks are in order. The relation (4.2) (in the particular case $\mathcal{H} \cong \mathbb{C}^{2}$ ) was the starting point for Baxter in his solution of the 8-vertex and $X Y Z$ models [1]. This is what he called the "pair-propagation through a vertex" property. In the context of the quantum inverse scattering method [2] the equivalent condition (4.3) is more customary. It defines local vacua of the (gauge-transformed) L-operator. A generalization of that solution to the higher spin $X Y Z$ model was given by Takebe in [12]. An algebro-geometric approach to the equation (4.2) for finite-dimensional matrices $\mathbf{L}_{i k}$ was developed by Krichever [21]. 
For L-operators with elliptic spectral parameter it is convenient to pass to the elliptic parametrization of the components of the vectors $\phi, \psi$. We introduce the 2-component vector

$$
|\zeta\rangle=\left(\begin{array}{c}
\bar{\theta}_{4}(\zeta) \\
\bar{\theta}_{3}(\zeta)
\end{array}\right)
$$

and the corresponding covector $\langle\zeta|=\left(\bar{\theta}_{4}(\zeta), \bar{\theta}_{3}(\zeta)\right)$ (recall that $\left.\bar{\theta}_{a}(\zeta)=\theta_{a}\left(\zeta \mid \frac{\tau}{2}\right)\right)$. The vector orthogonal to $\langle\zeta|$ is $|\zeta\rangle^{\perp}=\left(\begin{array}{r}\bar{\theta}_{3}(\zeta) \\ -\bar{\theta}_{4}(\zeta)\end{array}\right),\langle\zeta \mid \zeta\rangle^{\perp}=0$. More generally, we have:

$$
\langle\xi \mid \zeta\rangle^{\perp}=2 \theta_{1}(\xi+\zeta) \theta_{1}(\xi-\zeta)
$$

Note also that

$$
\left|\zeta+\frac{1}{2}(1+\tau)\right\rangle=e^{-\frac{\pi i \tau}{2}-2 \pi i \zeta}|\zeta\rangle^{\perp}
$$

Let us specify the general definition of the vacuum vectors for the elliptic L-operator (2.1). Writing $\mathrm{L}(\lambda)|\zeta\rangle$ (respectively, $\langle\zeta| \mathrm{L}(\lambda)$ ) we mean that the $2 \times 2$ matrix (2.1) acts on the 2-component vector from the left (respectively, on the 2-component covector from the right), as usual. Similarly, we introduce right and left vacuum vectors $X_{R}, X_{L}$ according to the relations

$$
\langle\zeta| \mathrm{L}(\lambda) X_{R}=\langle\xi| X_{R}^{\prime}, \quad X_{L}\langle\zeta| \mathrm{L}(\lambda)=X_{L}^{\prime}\langle\xi| .
$$

In the latter formula the difference operators $\mathbf{s}_{a}$ act on $X_{L}$ from the right.

The following pictorial representation is helpful:

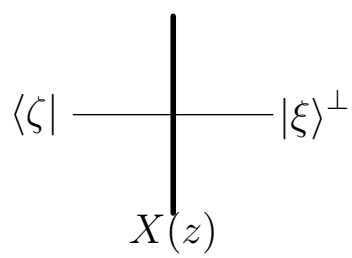

Here we use the standard conventions of the quantum inverse scattering method.

Introducing the operator

$$
\mathbf{K}=\mathbf{K}(\zeta, \xi)=\langle\zeta|\mathrm{L}(\lambda)| \xi\rangle^{\perp}
$$

we can rewrite (4.6) as $\mathbf{K} X_{R}=X_{L} \mathbf{K}=0$. The explicit form of the operator $\mathbf{K}$ is easily found from $(3.2)$ :

$$
\mathbf{K}=\mathbf{K}(\zeta, \xi)=\rho(z) e^{\eta \partial_{z}}+\rho(-z) e^{-\eta \partial_{z}},
$$

where

$$
\rho(z)=\frac{1}{\theta_{1}(2 z)} \prod_{\epsilon= \pm} \theta_{1}\left(z+\epsilon \zeta-\lambda_{+}\right) \theta_{1}\left(z+\epsilon \xi+\lambda_{-}\right) .
$$

So, the equations for the right and left vacuum vectors read

$$
\begin{gathered}
\rho(z) X_{R}(z+\eta)=-\rho(-z) X_{R}(z-\eta), \\
\rho(-z-\eta) X_{L}(z+\eta)=-\rho(z-\eta) X_{L}(z-\eta) .
\end{gathered}
$$

These equations themselves are not yet enough. An important additional input is the space where to solve them. Our choice, justified by the final results, is the following. We 
are going to solve the first equation in the space of combs finite from the left, $\mathcal{C}^{\vdash}$, while the second equation is to be solved in the space of combs finite from the right, $\mathcal{C}^{-}$. (For precise definitions of these spaces see Sec.3.)

Let us begin by solving equations (4.9), (4.10) in the space of meromorphic functions of $z$. This is easy to do in terms of the elliptic gamma-function $\Gamma(z \mid \tau, 2 \eta)$ introduced by Ruijsenaars [18] and recently studied in detail by Felder and Varchenko [19]. This function was introduced just to solve equations of such a type. Its main properties are summarized in Appendix A. In what follows we write $\Gamma(z \mid \tau, 2 \eta)=\Gamma(z)$ for brevity. Let $\mathcal{X}_{R}(z), \mathcal{X}_{L}(z)$ be meromorphic solutions of eqs. (4.9), (4.10), respectively. Using (A9), we easily find:

$$
\begin{gathered}
\mathcal{X}_{R}(z)=e^{-4 \pi i \ell z} \omega(\lambda, \zeta-\xi) \prod_{\epsilon= \pm} \frac{\Gamma\left(z+\epsilon \zeta+\lambda_{+}+\eta\right) \Gamma\left(z+\epsilon \xi-\lambda_{-}+\eta\right)}{\Gamma\left(z+\epsilon \zeta-\lambda_{+}+\eta\right) \Gamma\left(z+\epsilon \xi+\lambda_{-}+\eta\right)}, \\
\mathcal{X}_{L}(z)=e^{4 \pi i(\ell+1) z} \Phi_{\ell}(2 \lambda) \theta_{1}(2 z) \prod_{\epsilon= \pm} \frac{\Gamma\left(z+\epsilon \zeta-\lambda_{+}\right) \Gamma\left(z+\epsilon \xi+\lambda_{-}\right)}{\Gamma\left(z+\epsilon \zeta+\lambda_{+}+2 \eta\right) \Gamma\left(z+\epsilon \xi-\lambda_{-}+2 \eta\right)} .
\end{gathered}
$$

The $z$-independent normalization factors $\omega(\lambda, \zeta-\xi)$ in (4.11) and $\Phi_{\ell}(2 \lambda)$ in (4.12) are introduced here for later convenience. The function $\omega(\lambda, \zeta)$ is given by

$$
\omega(\lambda, \zeta)=\frac{\theta_{1}(2 \lambda-2 \ell \eta \mid 2 \eta)}{\theta_{1}(2 \lambda+\zeta \mid 2 \eta)} \exp \left(-\frac{\pi i \zeta^{2}}{2 \eta}-4 \pi i \ell \lambda\right)
$$

(the $\theta$-functions here have the modular parameter $2 \eta$ rather than $\tau$ !). The function $\Phi_{\ell}$ obeys the difference equation $\theta_{1}(z-2 \ell \eta) \Phi_{\ell}(z+2 \eta)=\theta_{1}(z+2(\ell+1) \eta) \Phi_{\ell}(z)$. For $\ell \in \frac{1}{2} \mathbb{Z}_{+}$ the solution is very simple:

$$
\Phi_{\ell}(z)=\prod_{k=-\ell}^{\ell} \theta_{1}(z+2 k \eta)
$$

where $k$ ranges over $-\ell,-\ell+1, \ldots, \ell$. For arbitrary complex values of $\ell$ the function $\Phi_{\ell}$ can be defined through the elliptic gamma-function (see (A16)). If necessary, we write $\mathcal{X}_{R, L}(z)=\mathcal{X}_{R, L}(z ; \zeta, \xi, \lambda)$. Note that $\mathcal{X}_{R, L}(z ; \zeta, \xi, \lambda)$ differs from $\mathcal{X}_{R, L}(z ; \xi, \zeta,-\lambda)$ only by a $z$-independent factor.

An important remark is in order. The solutions (4.11), (4.12) are by no means unique. Clearly, one can multiply them by an arbitrary function $f(z)$ such that $f(z+\eta)=f(z-\eta)$. In this paper, we do not take care of such $2 \eta$-periodic factors fixing them to be constant. In other words, (4.11), (4.12) are "minimal" solutions to eqs. (4.9), (4.10) in the sense of 18]. By this we break the symmetry between the three periods $1,2 \eta, \tau$ involved in the problem. In particular, the modular symmetry is broken since $2 \eta$-periodic factors may be important for good modular properties of the solutions. Indeed, the modular transformation of the elliptic gamma-function has the form [19]:

$$
\Gamma(z \mid \tau, 2 \eta)=e^{i \pi P(z)} \frac{\Gamma\left(\frac{z}{\tau} \mid-\frac{1}{\tau}, \frac{2 \eta}{\tau}\right)}{\Gamma\left(\frac{z-\tau}{2 \eta} \mid-\frac{1}{2 \eta}, \frac{\tau}{2 \eta}\right)},
$$

where $P(z)$ is a polynomial of third degree (see (A12)). The denominator is a $2 \eta$-periodic function which does not enter the minimal solution of the modular transformed equation. We note in passing that this formula is an elliptic version of eq. (40) from the paper [22], where a modular double of quantum group was introduced. We hope to discuss this point elsewhere. See also the end of Sec. 8. 
Now we can proceed to built a half-infinite comb solution. Any infinite comb of the form $X(z)=\mathcal{X}_{R, L}(z) \sum_{k \in \mathbb{Z}} \delta\left(z-z_{0}+2 k \eta\right)$ automatically satisfies eq. (4.9) (resp., (4.10)). How to truncate the comb holding these equations valid? Let $\mathcal{X}(z)$ be any one of $\mathcal{X}_{R}(z)$, $\mathcal{X}_{L}(z)$. The position of zeros and poles of the $\Gamma$-function suggests two possibilities:

(1) To set $z_{0}$ to be a zero of the function $\mathcal{X}: \mathcal{X}\left(z_{0}\right)=0$. If this zero comes from a pole of one of the $\Gamma$-functions downstairs, then $\mathcal{X}\left(z_{0}-2 k \eta\right)=0, k \geq 0$, and the comb is truncating from the left. If $z_{0}$ is a zero of one of the $\Gamma$-functions upstairs, then $\mathcal{X}\left(z_{0}-2 k \eta\right)=0, k<0$, and the comb is truncating from the right.

(2) To set $z_{0}$ to be a pole of $\mathcal{X}$ and define $X(z)=\sum_{k \in \mathbb{Z}}$ res $\left.\right|_{z=z_{0}-2 k \eta} \mathcal{X}(z) \delta\left(z-z_{0}+2 k \eta\right)$. If $z_{0}$ is a pole of a $\Gamma$-function upstairs, then the comb is truncating from the right. If the pole comes from a zero of one of the $\Gamma$-functions downstairs, then the comb is truncating from the left.

In the sequel we use both possibilities: (1) for $X_{R}$ and (2) for $X_{L}$. Other options are to be further analysed.

Assume that the parameters are in general position, i.e., there is no cancellation of zeros and poles in (4.11). (For $\ell \in \frac{1}{2} \mathbb{Z}_{+}$the sufficient conditions are $\lambda \neq 2 m \eta, \xi-\zeta \neq 2 m \eta$, $m \in \mathbb{Z}$.) Then $\mathcal{X}_{R}\left(\xi-\lambda_{-}-\eta-2 k \eta\right)=0$ for all integer $k \geq 0$. At the same time this function is not identically zero when $k$ is negative. Therefore,

$$
X_{R}^{+}(z ; \zeta, \xi, \lambda)=\mathcal{X}_{R}(z ; \zeta, \xi, \lambda) \sum_{k=1}^{\infty} \delta\left(z-\xi+\lambda_{-}+\eta-2 k \eta\right)
$$

is a non-trivial comb finite from the left which solves eq. (4.9). Clearly, this is not the only possibility to truncate the infinite comb from the left, even if to stick to (1). There are three other series of zeros of the function (4.11) coming from poles of the gamma-functions in the denominator. Any one of them can be used to generate, in the same way, a halfinfinite comb solution that belongs to $\mathcal{C}^{\vdash}$. However, inasmuch as the vacuum vectors are going to be kernels of difference operators, we want the $\delta$-functions in the vacuum vectors to depend on the differences $z-\zeta$ or $z-\xi$. So, along with (4.15), we have another comb obeying eq. (4.9):

$$
X_{R}^{-}(z ; \zeta, \xi, \lambda)=\mathcal{X}_{R}(z ; \xi, \zeta,-\lambda) \sum_{k=1}^{\infty} \delta\left(z-\zeta-\lambda_{+}+\eta-2 k \eta\right) .
$$

For $X_{L}$ we use $(2): \mathcal{X}_{L}(z)$ has poles at $z=\zeta+\lambda_{+}-2 k \eta, k \geq 0$. In general position, these poles are all simple. The lattice of poles is finite from the right. Therefore, we can construct a solution to eq. (4.10) in the space of combs finite from the right as follows:

$$
X_{L}^{+}(\lambda, \zeta, \xi ; z)=\left.\sum_{k=0}^{\infty} \operatorname{res}\right|_{z=\zeta+\lambda_{+}-2 k \eta} \mathcal{X}_{L}(z ; \zeta, \xi, \lambda) \delta\left(z-\zeta-\lambda_{+}+2 k \eta\right)
$$

Similarly to the case of the right vacuum vectors, we have a second solution:

$$
X_{L}^{-}(\lambda, \zeta, \xi ; z)=\left.\sum_{k=0}^{\infty} \operatorname{res}\right|_{z=\xi-\lambda_{-}-2 k \eta} \mathcal{X}_{L}(z ; \xi, \zeta,-\lambda) \delta\left(z-\xi+\lambda_{-}+2 k \eta\right) .
$$


To make these formulas closer to (4.15), (4.16), we introduce the auxiliary function

$$
\begin{gathered}
\mathcal{X}_{L}^{\varepsilon}(z ; \zeta, \xi, \lambda)=e^{4 \pi i(\ell+1) z} \Phi_{\ell}(2 \lambda) \theta_{1}(2 z) \times \\
\times \frac{\Gamma\left(z-\zeta-\lambda_{+}+\varepsilon\right) \Gamma\left(z+\zeta-\lambda_{+}\right) \Gamma\left(z-\xi+\lambda_{-}+\varepsilon\right) \Gamma\left(z+\xi+\lambda_{-}\right)}{\Gamma\left(z-\zeta+\lambda_{+}+2 \eta\right) \Gamma\left(z+\zeta+\lambda_{+}+2 \eta\right) \Gamma\left(z-\xi-\lambda_{-}+2 \eta\right) \Gamma\left(z+\xi-\lambda_{-}+2 \eta\right)},
\end{gathered}
$$

then, up to an irrelevant constant factor,

$$
\begin{gathered}
X_{L}^{+}(z ; \zeta, \xi, \lambda)=\lim _{\varepsilon \rightarrow 0} \varepsilon \mathcal{X}_{L}^{\varepsilon}(z ; \zeta, \xi, \lambda) \sum_{k=0}^{\infty} \delta\left(z-\zeta-\lambda_{+}+2 k \eta\right), \\
X_{L}^{-}(\lambda, \zeta, \xi ; z)=\lim _{\varepsilon \rightarrow 0} \varepsilon \mathcal{X}_{L}^{\varepsilon}(z ; \xi, \zeta,-\lambda) \sum_{k=0}^{\infty} \delta\left(z-\xi+\lambda_{-}+2 k \eta\right) .
\end{gathered}
$$

Here one should first substitute the values of $z$ in the $\delta$-functions and then take the limit.

Note that the function $\rho(z)$ and the meromorphic solutions $\mathcal{X}_{R, L}$ are invariant with respect to the simultaneous change $\lambda \rightarrow-\lambda, \zeta \leftrightarrow \xi$ while the half-infinite combs $X_{R, L}^{ \pm}$are transformed one into another:

$$
\begin{aligned}
& X_{R}^{-}(z ; \zeta, \xi, \lambda)=X_{R}^{+}(z ; \xi, \zeta,-\lambda), \\
& X_{L}^{-}(\lambda, \zeta, \xi ; z)=X_{L}^{+}(-\lambda, \xi, \zeta ; z) .
\end{aligned}
$$

The construction of local vacuum vectors is completed. The vacuum vectors are defined up to an arbitrary normalization factor which may depend on $\zeta, \xi$ and $\lambda$. In our normalization, the final formulas have the most simple form.

By a straightforward computation, which uses some standard identities for thetafunctions, one can prove the following relations valid for $X_{R, L}^{ \pm}=X_{R, L}$ :

$$
\begin{aligned}
& \langle\zeta| \mathrm{L}(\lambda) X_{R}(z ; \zeta, \xi, \lambda)=\theta_{1}\left(2 \lambda_{-}\right)\langle\xi| X_{R}(z ; \zeta, \xi, \lambda+\eta), \\
& X_{L}(\zeta, \xi, \lambda ; z)\langle\zeta| \mathrm{L}(\lambda)=\theta_{1}\left(2 \lambda_{+}\right) X_{L}(\zeta, \xi, \lambda-\eta ; z)\langle\xi|,
\end{aligned}
$$

which are simply (4.6) in the specified parametrization. In the first equation, each operator entry of the matrix $L(\lambda)$ acts on the vacuum vector from the left while in the second one they act from the right. In the both equations, the two-component covector $\langle\zeta|$ is applied from the left. Using (3.3) and (4.5), one can represent these equations in the form, where the two-component vector is applied from the right:

$$
\begin{aligned}
& \mathrm{L}(\lambda)|\xi\rangle^{\perp} X_{R}(z ; \zeta, \xi, \lambda)=\theta_{1}\left(2 \lambda_{+}\right)|\zeta\rangle^{\perp} X_{R}(z ; \zeta, \xi, \lambda-\eta), \\
& X_{L}(\zeta, \xi, \lambda ; z) \mathrm{L}(\lambda)|\xi\rangle^{\perp}=\theta_{1}\left(2 \lambda_{-}\right)|\zeta\rangle^{\perp} X_{L}(\zeta, \xi, \lambda+\eta ; z) .
\end{aligned}
$$

For the holomorphic vacuum vectors $\left(\right.$ (4.11) at $\left.\ell \in \mathbb{Z}_{+}\right)$these relations hold [12] under the additional restriction $\xi-\zeta \in 2 \eta \mathbb{Z}$. 


\section{Global vacuum vectors}

The next step, in accordance with [1]], is to find the vacuum vectors for each $L^{i}(\lambda)$ and consider the action of the transfer matrix $\mathbf{T}(\lambda)(2.3)$ on their direct product along the chain. It then appears that the result of this action has a very special and simple form: it is a sum of two terms, each of them being a vacuum vector that belongs to the same family. From the technical point of view, the easiest way to see this is to use the gauge transformation argument, now standard.

Let us make the "gauge transformation" of the L-operator:

$$
\stackrel{i}{\mathrm{~L}}(\lambda) \longrightarrow G\left(\zeta_{i}\right) \stackrel{i}{\mathrm{~L}}(\lambda)\left(G\left(\zeta_{i+1}\right)\right)^{-1}=\stackrel{i}{\prime}^{\prime}(\lambda),
$$

where $G(\zeta)$ are some $2 \times 2 c$-number matrices depending on the parameter $\zeta$. If $\zeta_{N+1}=\zeta_{1}$, then the trace is not changed:

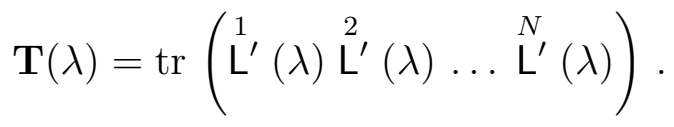

We set the matrix $G(\zeta)$ to be

$$
G(\zeta)=\left(\begin{array}{cc}
\bar{\theta}_{4}(\zeta) & \bar{\theta}_{3}(\zeta) \\
0 & 1
\end{array}\right)
$$

Then, acting on a vacuum vector $X_{R}$ by each element of the gauge-transformed L-operator and using the relations (4.22), (4.24), we obtain:

$$
\begin{aligned}
& \left(\begin{array}{ll}
\mathbf{L}_{11}^{\prime} X_{R} & \mathbf{L}_{12}^{\prime} X_{R} \\
\mathbf{L}_{21}^{\prime} X_{R} & \mathbf{L}_{22}^{\prime} X_{R}
\end{array}\right)= \\
& =\frac{1}{\bar{\theta}_{4}(\xi)}\left(\begin{array}{cc}
\bar{\theta}_{4}(\zeta) & \bar{\theta}_{3}(\zeta) \\
0 & 1
\end{array}\right)\left(\begin{array}{ll}
\mathbf{L}_{11} X_{R} & \mathbf{L}_{12} X_{R} \\
\mathbf{L}_{21} X_{R} & \mathbf{L}_{22} X_{R}
\end{array}\right)\left(\begin{array}{cr}
1 & -\bar{\theta}_{3}(\xi) \\
0 & \bar{\theta}_{4}(\xi)
\end{array}\right)= \\
& =\left(\begin{array}{cc}
X_{R}^{\prime} & 0 \\
& \frac{\bar{\theta}_{4}(\zeta)}{\bar{\theta}_{4}(\xi)} X_{R}^{\prime \prime}
\end{array}\right) .
\end{aligned}
$$

Here $X_{R}^{\prime}=\theta_{1}\left(2 \lambda_{-}\right) X_{R}\left(z_{i} ; \zeta, \xi, \lambda+\eta\right), X_{R}^{\prime \prime}=\theta_{1}\left(2 \lambda_{+}\right) X_{R}\left(z_{i} ; \zeta, \xi, \lambda-\eta\right)$ and we imply that $\zeta_{i}=\zeta, \zeta_{i+1}=\xi$. The star in the left-down corner stands for something irrelevant for us.

We define the global vacuum vectors $\vec{X}_{R, L}^{ \pm}$to be products of the local ones over all sites of the chain:

$$
\begin{aligned}
& \vec{X}_{R}^{ \pm}(\lambda)=\vec{X}_{R}^{ \pm}(\vec{z}, \vec{\zeta}, \lambda)=\prod_{i=1}^{N} X_{R}^{ \pm}\left(z_{i} ; \zeta_{i}, \zeta_{i+1}, \lambda\right), \\
& \vec{X}_{L}^{ \pm}(\lambda)=\vec{X}_{L}^{ \pm}(\lambda, \vec{\zeta}, \vec{z})=\prod_{i=1}^{N} X_{L}^{ \pm}\left(\lambda, \zeta_{i}, \zeta_{i+1} ; z_{i}\right),
\end{aligned}
$$

where the notation $\vec{z}=\left\{z_{1}, \ldots, z_{N}\right\}, \vec{\zeta}=\left\{\zeta_{1}, \ldots, \zeta_{N}\right\}$ is used. The parameters $\zeta_{i}$ are naturally asociated with the horizontal edges of the lattice: 


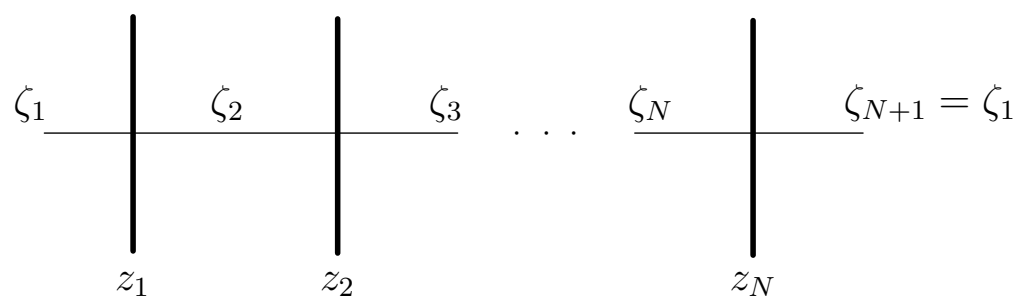

Then

$$
\mathbf{T}(\lambda) \vec{X}_{R}^{ \pm}(\lambda)=\operatorname{tr} \prod_{i=1}^{N}\left(\mathrm{~L}^{i}(\lambda) X_{R}^{ \pm}\left(z_{i} ; \zeta_{i}, \zeta_{i+1}, \lambda\right)\right)
$$

and similarly for the $\vec{X}_{L}^{ \pm}(\lambda)$. The triangularity of the matrix (5.3) allows one to compute the trace. Taking into account the periodic boundary condition, we arrive at the following relations:

$$
\begin{aligned}
& \mathbf{T}(\lambda) \vec{X}_{R}^{ \pm}(\lambda)=\theta_{1}^{N}\left(2 \lambda_{-}\right) \vec{X}_{R}^{ \pm}(\lambda+\eta)+\theta_{1}^{N}\left(2 \lambda_{+}\right) \vec{X}_{R}^{ \pm}(\lambda-\eta), \\
& \vec{X}_{L}^{ \pm}(\lambda) \mathbf{T}(\lambda)=\theta_{1}^{N}\left(2 \lambda_{-}\right) \vec{X}_{L}^{ \pm}(\lambda+\eta)+\theta_{1}^{N}\left(2 \lambda_{+}\right) \vec{X}_{L}^{ \pm}(\lambda-\eta) .
\end{aligned}
$$

Let us introduce left and right $Q$-operators, $\mathbf{Q}_{L}^{ \pm}$and $\mathbf{Q}_{R}^{ \pm}$(the pre-Q-operators). They are difference operators acting on functions $f\left(z_{1}, \ldots, z_{N}\right)$ of $N$ variables, respectively from the left and from the right, as follows:

$$
\begin{aligned}
& \left(\mathbf{Q}_{L}^{ \pm} f\right)(\vec{\zeta})=\int d \vec{z} \vec{X}_{L}^{ \pm}(\lambda, \vec{\zeta}, \vec{z}) f(\vec{z}), \\
& \left(f \mathbf{Q}_{R}^{ \pm}\right)(\vec{\zeta})=\int d \vec{z} f(\vec{z}) \vec{X}_{R}^{ \pm}(\vec{z}, \vec{\zeta}, \lambda) .
\end{aligned}
$$

The kernels here are the global vacuum vectors defined above. The symbol $\int d \vec{z}=$ $\int \ldots \int d z_{1} \ldots d z_{N}$ means the natural pairing in the tensor product of the local functional spaces. On each factor, this pairing coincides with (3.8).

The formulas (5.6), (5.7) can be written as the operator relations:

$$
\begin{aligned}
& \mathbf{T}(\lambda) \mathbf{Q}_{R}^{ \pm}(\lambda)=\theta_{1}^{N}\left(2 \lambda_{-}\right) \mathbf{Q}_{R}^{ \pm}(\lambda+\eta)+\theta_{1}^{N}\left(2 \lambda_{+}\right) \mathbf{Q}_{R}^{ \pm}(\lambda-\eta), \\
& \mathbf{Q}_{L}^{ \pm}(\lambda) \mathbf{T}(\lambda)=\theta_{1}^{N}\left(2 \lambda_{-}\right) \mathbf{Q}_{L}^{ \pm}(\lambda+\eta)+\theta_{1}^{N}\left(2 \lambda_{+}\right) \mathbf{Q}_{L}^{ \pm}(\lambda-\eta) .
\end{aligned}
$$

One might multiply the pre- $Q$-operators by a scalar normalization factor $f(\lambda)$ such that $f(\lambda)=f(\lambda+\eta)$. Clearly, these relations remain unchanged.

\section{Basic commutation relations}

In this section we prove the basic commutation relations

$$
\begin{aligned}
& \mathbf{Q}_{L}^{ \pm}(\lambda) \mathbf{Q}_{R}^{ \pm}\left(\lambda^{\prime}\right)=\mathbf{Q}_{L}^{ \pm}\left(\lambda^{\prime}\right) \mathbf{Q}_{R}^{ \pm}(\lambda), \\
& \mathbf{Q}_{L}^{+}(\lambda) \mathbf{Q}_{R}^{-}\left(\lambda^{\prime}\right)=\mathbf{Q}_{L}^{-}\left(\lambda^{\prime}\right) \mathbf{Q}_{R}^{+}(\lambda) .
\end{aligned}
$$

Let us start from the proof of (6.1) for $\mathbf{Q}_{L, R}^{+}$. To this end, consider the function

$$
\int d^{N} \vec{z} \vec{X}_{L}^{+}(\lambda, \vec{\zeta}, \vec{z}) \vec{X}_{R}^{+}\left(\vec{z}, \vec{\zeta}^{\prime}, \lambda^{\prime}\right)=\prod_{i=1}^{N} \int d z_{i} X_{L}^{+}\left(\lambda, \zeta_{i}, \zeta_{i+1} ; z_{i}\right) X_{R}^{+}\left(z_{i} ; \zeta_{i}^{\prime}, \zeta_{i+1}^{\prime}, \lambda^{\prime}\right)
$$


which is the kernel of the operator product in the left hand side of (6.1). Schematically, the structure of the kernel can be illustrated as follows:

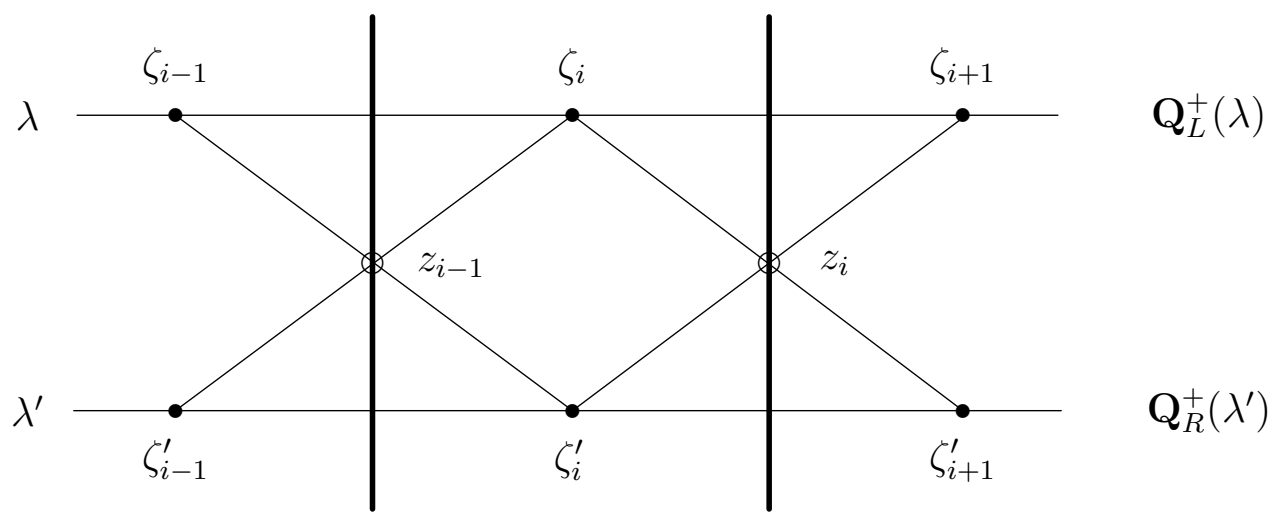

As is clear from (6.3), the kernel is a product of local multipliers associated with each pair $(i, i+1)$ of adjacent sites of the chain. For a while, we set $\zeta_{i}=\zeta, \zeta_{i+1}=\xi$. Therefore, we should investigate the function

$$
F_{\zeta^{\prime} \xi^{\prime}}^{\zeta \xi}\left(\lambda, \lambda^{\prime}\right)=\int d z X_{L}^{+}(\lambda, \zeta, \xi ; z) X_{R}^{+}\left(z ; \zeta^{\prime}, \xi^{\prime}, \lambda^{\prime}\right)
$$

Let us begin with the $X_{L}^{+}$. Up to an irrelevant constant factor, (4.19) reads

$$
X_{L}^{+}(\lambda, \zeta, \xi ; z)=\lim _{\varepsilon \rightarrow 0}\left[\varepsilon \mathcal{X}_{L}^{\varepsilon}\left(\zeta+\lambda_{+} ; \zeta, \xi, \lambda\right)\right] \sum_{k \geq 0} x_{k}(\zeta, \xi, \lambda) \delta\left(z-\zeta-\lambda_{+}+2 k \eta\right),
$$

where

$$
\begin{aligned}
& x_{k}(\zeta, \xi, \lambda)=\frac{1}{[k] !} \frac{\theta_{1}\left(2 \zeta+2 \lambda_{+}-4 k \eta\right)}{\theta_{1}\left(2 \zeta+2 \lambda_{+}\right)} \times \\
\times & \prod_{j=0}^{k-1} \frac{\theta_{1}\left(-2 \lambda_{+}+2 j \eta\right) \theta_{1}\left(-2 \lambda_{+}-2 \zeta+2 j \eta\right) \theta_{1}(\xi-\zeta-2 \ell \eta+2 j \eta) \theta_{1}(-\xi-\zeta-2 \ell \eta+2 j \eta)}{\theta_{1}(-2 \zeta+2(j+1) \eta) \theta_{1}(\xi-\zeta-2 \lambda+2(j+1) \eta) \theta_{1}(-\xi-\zeta-2 \lambda+2(j+1) \eta)} .
\end{aligned}
$$

Substituting (6.5) and $X_{R}^{+}\left(z ; \zeta^{\prime}, \xi^{\prime}, \lambda^{\prime}\right)=\mathcal{X}_{R}\left(z ; \zeta^{\prime}, \xi^{\prime}, \lambda^{\prime}\right) \sum_{k^{\prime} \geq 0} \delta\left(z-\xi^{\prime}+\lambda_{-}^{\prime}-\eta-2 k^{\prime} \eta\right.$ ) (see (4.15)) into (6.4), performing the $z$-integral according to the pairing rule (3.9), and passing to summation over $n=k+k^{\prime} \geq 0$, we get:

$$
\begin{gathered}
F_{\zeta^{\prime} \xi^{\prime}}^{\zeta \xi}\left(\lambda, \lambda^{\prime}\right)=\lim _{\varepsilon \rightarrow 0}\left[\varepsilon \mathcal{X}_{L}^{\varepsilon}\left(\zeta+\lambda_{+} ; \zeta, \xi, \lambda\right)\right] \times \\
\times \sum_{n \geq 0} \delta\left(\zeta-\xi^{\prime}+\lambda+\lambda^{\prime}-\eta-2 n \eta\right) \sum_{k=0}^{n} x_{k}(\zeta, \xi, \lambda) \mathcal{X}_{R}\left(\zeta+\lambda_{+}-2 k \eta ; \zeta^{\prime}, \xi^{\prime}, \lambda^{\prime}\right) .
\end{gathered}
$$

The next step is to identify the finite sum over $k$ with the terminating modular hypergeometric function ${ }_{10} W_{9}$. For the definition see Appendix A. Plugging here the explicit formula for $\mathcal{X}_{R}$ (4.11) and using (A13), one can represent (6.7) in the form

$$
\begin{aligned}
F_{\zeta^{\prime} \xi^{\prime}}^{\zeta \xi}\left(\lambda, \lambda^{\prime}\right) & =F_{\operatorname{sym}}^{\zeta \xi, \zeta^{\prime} \xi^{\prime}}\left(\lambda, \lambda^{\prime}\right) \omega\left(\lambda^{\prime}, \zeta^{\prime}-\xi^{\prime}\right) F_{1}^{\zeta \xi}(\lambda) F_{2}^{\zeta \zeta^{\prime} \xi^{\prime}}\left(\lambda, \lambda^{\prime}\right) \frac{g_{\xi \xi^{\prime}}\left(\lambda-\lambda^{\prime}\right)}{g_{\zeta \zeta^{\prime}}\left(\lambda-\lambda^{\prime}\right)} \times \\
& \times \sum_{n \geq 0} 10 W_{9}\left(\alpha_{1} ; \alpha_{4}, \ldots, \alpha_{9},-n\right) \delta\left(\zeta-\xi^{\prime}+\lambda+\lambda^{\prime}-\eta-2 n \eta\right),
\end{aligned}
$$


where

$$
F_{\mathrm{sym}}^{\zeta \xi, \zeta^{\prime} \xi^{\prime}}=\frac{e^{2 \pi i \zeta} \Gamma(2 \zeta)}{\Gamma(\zeta-\xi+2(\ell+1) \eta) \Gamma(\zeta+\xi+2(\ell+1) \eta)} \prod_{\epsilon= \pm} \frac{\Gamma\left(\zeta+\epsilon \zeta^{\prime}+\lambda_{+}+\lambda_{+}^{\prime}+\eta\right)}{\Gamma\left(\zeta+\epsilon \xi^{\prime}+\lambda+\lambda^{\prime}+\eta\right)}
$$

is a symmetric function of $\lambda, \lambda^{\prime}$ for all $\zeta, \xi, \zeta^{\prime}, \xi^{\prime}, \omega\left(\lambda^{\prime}, \zeta^{\prime}-\xi^{\prime}\right)$ is defined in (4.13), and

$$
\begin{gathered}
g_{\zeta \zeta^{\prime}}(\lambda)=\Gamma\left(\zeta+\zeta^{\prime}+\lambda+\eta\right) \Gamma\left(\zeta-\zeta^{\prime}+\lambda+\eta\right) \\
F_{1}^{\zeta \xi}(\lambda)=e^{-4 \pi i \ell \lambda} \frac{\Gamma(\zeta-\xi+2 \lambda) \Gamma(\zeta+\xi+2 \lambda)}{\Gamma\left(2 \lambda_{-}\right) \Gamma\left(2 \zeta+2 \lambda_{+}\right)} \\
F_{2}^{\zeta \zeta^{\prime} \xi^{\prime}}\left(\lambda, \lambda^{\prime}\right)=\frac{\Gamma\left(\zeta+\xi^{\prime}+\lambda_{+}-\lambda_{-}^{\prime}+\eta\right) \Gamma\left(\zeta-\xi^{\prime}+\lambda_{+}-\lambda_{-}^{\prime}+\eta\right)}{\Gamma\left(\xi+\xi^{\prime}+\lambda-\lambda^{\prime}+\eta\right) \Gamma\left(\xi-\xi^{\prime}+\lambda-\lambda^{\prime}+\eta\right)} .
\end{gathered}
$$

The function ${ }_{10} W_{9}$ is defined in (A20). It is an elliptic analog of the very-well poised hypergeometric series. The values of parameters are as follows:

$$
\begin{aligned}
& -2 \eta \alpha_{1}=2 \zeta+2 \lambda+2 \ell \eta \quad-2 \eta \alpha_{7}=\zeta+\xi+2 \ell \eta \\
& -2 \eta \alpha_{4}=2 \lambda+2 \ell \eta \quad-2 \eta \alpha_{8}=\zeta-\xi+2 \ell \eta \\
& -2 \eta \alpha_{5}=\zeta+\zeta^{\prime}+\lambda-\lambda^{\prime}-\eta \quad-2 \eta \alpha_{9}=\zeta+\xi^{\prime}+\lambda+\lambda^{\prime}-\eta \\
& -2 \eta \alpha_{6}=\zeta-\zeta^{\prime}+\lambda-\lambda^{\prime}-\eta \quad-2 \eta \alpha_{10}=\zeta-\xi^{\prime}+\lambda+\lambda^{\prime}-\eta=2 n \eta
\end{aligned}
$$

Note that the parameters in the right column are symmetric under the permutation $\lambda \leftrightarrow \lambda^{\prime}$. The series is balanced that means that the balancing condition

$$
2+3 \alpha_{1}=\sum_{m=1}^{7} \alpha_{m+3}
$$

is satisfied. The series is terminating since $\alpha_{10}=-n$, where $n$ is a non-negative integer number.

Let us move the factor $F_{2}\left(\lambda, \lambda^{\prime}\right)$ inside the sum $\sum_{n \geq 0}$ and set $\xi^{\prime}=\zeta+\lambda+\lambda^{\prime}-\eta-2 n \eta$ in the $n$-th term (this is what the $\delta$-function suggests to do). After using (A13), (A14) and extracting the common factor

$$
F_{3}^{\zeta \xi}\left(\lambda, \lambda^{\prime}\right)=\frac{\Gamma\left(2 \zeta+2 \lambda_{+}\right) \Gamma\left(-2 \lambda_{+}^{\prime}+2 \eta\right)}{\Gamma(\zeta+\xi+2 \lambda) \Gamma\left(-\zeta+\xi-2 \lambda^{\prime}+2 \eta\right)},
$$

the sum over $n$ becomes equal to

$$
F_{3}^{\zeta \xi}\left(\lambda, \lambda^{\prime}\right) \sum_{n \geq 0} \frac{\left[\alpha_{1}-\alpha_{7}+1\right]_{n}\left[\beta_{1}-\alpha_{7}-\alpha_{8}+1\right]_{n}}{\left[\alpha_{1}+1\right]_{n}\left[\beta_{1}-\alpha_{8}+1\right]_{n}} W_{9}\left(\alpha_{1} ; \alpha_{4}, \ldots,-n\right) \delta\left(\zeta-\xi^{\prime}+\lambda+\lambda^{\prime}-\eta-2 n \eta\right)
$$

where we use the elliptic Pochhammer symbol $[x]_{n}=[x][x+1] \ldots[x+n-1]$, and $\beta_{1}=$ $2 \alpha_{1}+1-\alpha_{4}-\alpha_{5}-\alpha_{6}$. Now, we recall the elliptic analog (A21) of the Bailey transformation formula for the terminating balanced ${ }_{10} W_{9}$. It is easily seen from (6.12) that the Bailey transformation of the parameters $\alpha_{i} \rightarrow \beta_{i}$ (A22) is equivalent to the permutation $\lambda \leftrightarrow \lambda^{\prime}$ while all the other parameters remain unchanged. Therefore, as it follows from (A21), each term inside the sum over $n$ in (6.15) is symmetric under the permutation $\lambda \leftrightarrow \lambda^{\prime}$. 
The common factor $F_{3}$ is not symmetric. Combining it with the remaining factors and taking into account (A10), we obtain:

$$
\begin{gathered}
F_{\zeta^{\prime} \xi^{\prime}}^{\zeta \xi}\left(\lambda, \lambda^{\prime}\right)=\frac{g_{\xi \xi^{\prime}}\left(\lambda-\lambda^{\prime}\right)}{g_{\zeta \zeta^{\prime}}\left(\lambda-\lambda^{\prime}\right)} \times \\
\times \sum_{n \geq 0} \mathcal{F}_{\mathrm{sym}}^{\zeta \xi, \zeta^{\prime} \xi^{\prime}}\left(\lambda, \lambda^{\prime} ; n\right) \omega(\lambda, \zeta-\xi) \omega\left(\lambda^{\prime}, \zeta^{\prime}-\xi^{\prime}\right) \delta\left(\zeta-\xi^{\prime}+\lambda+\lambda^{\prime}-\eta-2 n \eta\right),
\end{gathered}
$$

where $\mathcal{F}_{\text {sym }}^{\zeta \xi, \zeta^{\prime} \xi^{\prime}}\left(\lambda, \lambda^{\prime} ; n\right)$ is symmetric under the permutation of $\lambda$ and $\lambda^{\prime}$ for all $n, \zeta, \xi, \zeta^{\prime}, \xi^{\prime}$, and the functions $g, \omega$ are given in (6.9), (4.13) respectively.

To obtain the kernel (6.3), we should set $\zeta=\zeta_{i}, \xi=\zeta_{i+1}$ and take the product of the local factors (6.16) over $i$ from 1 to $N$ provided $\zeta_{N+1}=\zeta_{1}$. Therefore, the ratios of the $g$-functions cancel. The only factor which is not manifestly symmetric under the permutation $\lambda \leftrightarrow \lambda^{\prime}$ comes from the product of the $\omega$-functions. This factor is equal to

$$
\exp \left(-\frac{\pi i}{2 \eta} \sum_{j=1}^{N}\left(\zeta_{j}^{\prime}-\zeta_{j+1}^{\prime}\right)^{2}\right) \prod_{i=1}^{N}\left(\theta_{1}\left(\zeta_{i}-\zeta_{i+1}-2 \lambda \mid 2 \eta\right) \theta_{1}\left(\zeta_{i}^{\prime}-\zeta_{i+1}^{\prime}-2 \lambda^{\prime} \mid 2 \eta\right)\right)^{-1}
$$

However, we should take into account the constraints imposed by the $\delta$-functions: $\zeta_{i}^{\prime}=$ $\zeta_{i+1}-\lambda-\lambda^{\prime}+\eta+2 n_{i-1} \eta$, where $n_{i} \in \mathbb{Z}$. In other words, (6.17) is zero unless $\zeta_{i}^{\prime}-\zeta_{i+1}^{\prime}=$ $\zeta_{i-1}-\zeta_{i}+2\left(n_{i-1}-n_{i}\right) \eta$. From the monodromy properties of the $\theta$-function it then follows that this function is symmetric, too. Therefore, the commutation relation (6.1) for $\mathbf{Q}_{L, R}^{+}$ is proved. To prove it for $\mathbf{Q}_{L, R}^{-}$, there is no need to repeat the calculations since $X_{L, R}^{-}$is related to $X_{L, R}^{+}$via (4.21).

The proof of (6.2) requires a similar but different calculation. Its scheme is outlined below. The kernel of the operator in the left hand side is

$$
\begin{aligned}
& \prod_{i=1}^{N} \int d z_{i} X_{L}^{+}\left(\lambda, \zeta_{i}, \zeta_{i+1} ; z_{i}\right) X_{R}^{-}\left(z_{i} ; \zeta_{i}^{\prime}, \zeta_{i+1}^{\prime}, \lambda^{\prime}\right)= \\
= & \prod_{i=1}^{N} \int d z_{i} X_{L}^{+}\left(\lambda, \zeta_{i}, \zeta_{i+1} ; z_{i}\right) X_{R}^{+}\left(z_{i} ; \zeta_{i+1}^{\prime}, \zeta_{i}^{\prime},-\lambda^{\prime}\right),
\end{aligned}
$$

where (4.21) is used. Again, set $\zeta_{i}=\zeta, \zeta_{i+1}=\xi, \zeta_{i}^{\prime}=\zeta^{\prime}, \zeta_{i+1}^{\prime}=\xi^{\prime}$, and examine the symmetry properties of the function

$$
\tilde{F}_{\zeta^{\prime} \xi^{\prime}}^{\zeta \xi}\left(\lambda, \lambda^{\prime}\right)=\int d z X_{L}^{+}(\lambda, \zeta, \xi ; z) X_{R}^{+}\left(z ; \xi, \zeta,-\lambda^{\prime}\right)
$$

under the simultaneous change $\zeta \leftrightarrow \xi, \zeta^{\prime} \leftrightarrow \xi^{\prime}, \lambda \rightarrow-\lambda^{\prime}, \lambda^{\prime} \rightarrow-\lambda$. It is enough to show that the product of such functions over the chain is invariant under this substitution in each factor. Proceeding as above, we obtain the representation of this function similar to (6.8):

$$
\begin{aligned}
\tilde{F}_{\zeta^{\prime} \xi^{\prime}}^{\zeta \xi}\left(\lambda, \lambda^{\prime}\right) & =F_{\operatorname{Sym}}^{\zeta \xi, \zeta^{\prime} \xi^{\prime}}\left(\lambda, \lambda^{\prime}\right) \omega\left(-\lambda^{\prime}, \xi^{\prime}-\zeta^{\prime}\right) F_{1}^{\zeta \xi}(\lambda) F_{2}^{\zeta \zeta^{\prime} \xi^{\prime}}\left(\lambda, \lambda^{\prime}\right) \frac{g_{\xi \xi^{\prime}}\left(\lambda-\lambda^{\prime}\right)}{g_{\zeta \zeta^{\prime}}\left(\lambda-\lambda^{\prime}\right)} \times \\
& \times \sum_{n \geq 0} 10 W_{9}\left(\tilde{\alpha}_{1} ; \tilde{\alpha}_{4}, \ldots, \tilde{\alpha}_{10}\right) \delta\left(\zeta-\zeta^{\prime}+\lambda-\lambda^{\prime}-\eta-2 n \eta\right)
\end{aligned}
$$


with the same functions $F_{\text {sym }}, \omega, F_{1}, F_{2}$, and $g$ as before. The parameters of the series ${ }_{10} W_{9}$ are also the same (see (6.12)); however, for the sake of convenience they are permuted in the following way: $\tilde{\alpha}_{1}=\alpha_{1}, \tilde{\alpha}_{4}=\alpha_{8}, \tilde{\alpha}_{5}=\alpha_{9}, \tilde{\alpha}_{6}=\alpha_{10}, \tilde{\alpha}_{7}=\alpha_{4}, \tilde{\alpha}_{8}=\alpha_{5}, \tilde{\alpha}_{9}=\alpha_{7}$, $\tilde{\alpha}_{10}=\alpha_{6}$. (Clearly, the series is symmetric under the permutation of all parameters except $\alpha_{1}$.) What differs is the argument of the $\delta$-function. Note that the series is terminating since the $\delta$-function is zero unless $\tilde{\alpha}_{10}=-n$. Next, we make the Bailey transformation (A21) passing from $\tilde{\alpha}_{i}$ to $\tilde{\beta}_{i}$ according to (A22). The result can be represented as a product of three factors:

$$
\tilde{F}_{\zeta^{\prime} \xi^{\prime}}^{\zeta \xi}\left(\lambda, \lambda^{\prime}\right)=A_{\zeta^{\prime} \xi^{\prime}}^{\zeta \xi}\left(\lambda, \lambda^{\prime}\right) \times\left[\begin{array}{l}
\text { invariant under } \zeta \leftrightarrow \xi, \\
\zeta^{\prime} \leftrightarrow \xi^{\prime}, \lambda \leftrightarrow-\lambda^{\prime}
\end{array}\right] \times\left[\begin{array}{l}
\text { factors that cancel in the } \\
\text { product over the chain }
\end{array}\right],
$$

where

$$
A_{\zeta^{\prime} \xi^{\prime}}^{\zeta \xi}\left(\lambda, \lambda^{\prime}\right)=\frac{\exp \left(-\pi i\left(\xi^{\prime}-\zeta^{\prime}\right)^{2} /(2 \eta)\right)}{\theta_{1}\left(\xi^{\prime}-\zeta^{\prime}-2 \lambda^{\prime} \mid 2 \eta\right) \theta_{1}(\zeta-\xi+2 \lambda \mid 2 \eta)} .
$$

The product $\prod_{i=1}^{N} A_{\zeta_{i}^{\prime} \zeta_{i+1}^{\prime}}^{\zeta_{i} \zeta_{i+1}}\left(\lambda, \lambda^{\prime}\right)$ is the only thing here which is not manifestly invariant under the simultaneous change $\zeta_{i} \leftrightarrow \zeta_{i+1}, \zeta_{i}^{\prime} \leftrightarrow \zeta_{i+1}^{\prime}, \lambda \leftrightarrow-\lambda^{\prime}$ in each factor. However, the same argument as the one used for (6.17) establishes the invariance.

\section{From pre- $Q$-operators to $Q$-operator}

Suppose the operators $\mathbf{Q}_{L}^{+}$and $\mathbf{Q}_{R}^{+}$are invertible at some particular $\lambda=\lambda_{*}$. Following [1], we define

$$
\mathbf{Q}^{ \pm}(\lambda)=\left(\mathbf{Q}_{L}^{+}\left(\lambda_{*}\right)\right)^{-1} \mathbf{Q}_{L}^{ \pm}(\lambda)=\mathbf{Q}_{R}^{ \pm}(\lambda)\left(\mathbf{Q}_{R}^{+}\left(\lambda_{*}\right)\right)^{-1}
$$

The second equality is due to the basic commutation relations (6.1), (6.2). It then follows, on applying (6.1), (6.2) once again, that $\left[\mathbf{Q}^{+}(\lambda), \mathbf{Q}^{+}(\mu)\right]=\left[\mathbf{Q}^{+}(\lambda), \mathbf{Q}^{-}(\mu)\right]=$ $\left[\mathbf{Q}^{-}(\lambda), \mathbf{Q}^{-}(\mu)\right]=0$ for all $\lambda, \mu$ :

$$
\begin{aligned}
& \mathbf{Q}^{+}(\lambda) \mathbf{Q}^{+}(\mu)= \\
= & \left(\mathbf{Q}_{L}^{+}\left(\lambda_{*}\right)\right)^{-1} \mathbf{Q}_{L}^{+}(\lambda) \mathbf{Q}_{R}^{+}(\mu)\left(\mathbf{Q}_{R}^{+}\left(\lambda_{*}\right)\right)^{-1}= \\
= & \left(\mathbf{Q}_{L}^{+}\left(\lambda_{*}\right)\right)^{-1} \mathbf{Q}_{L}^{+}(\mu) \mathbf{Q}_{R}^{+}(\lambda)\left(\mathbf{Q}_{R}^{+}\left(\lambda_{*}\right)\right)^{-1}= \\
= & \mathbf{Q}^{+}(\mu) \mathbf{Q}^{+}(\lambda),
\end{aligned}
$$

and similarly for $\mathbf{Q}^{-}$. Both equations (5.10), (5.11) lead to the Baxter $T-Q$ relation

$$
\mathbf{T}(\lambda) \mathbf{Q}(\lambda)=\theta_{1}^{N}(2 \lambda-2 \ell \eta) \mathbf{Q}(\lambda+\eta)+\theta_{1}^{N}(2 \lambda+2 \ell \eta) \mathbf{Q}(\lambda-\eta),
$$

where $\mathbf{Q}$ is any one of $\mathbf{Q}^{ \pm}$. Besides, it follows that $\mathbf{Q}^{ \pm}(\lambda)$ commute with $\mathbf{T}(\mu)$ for all $\lambda, \mu$.

Suppose $\ell \notin \mathbb{Z}_{+}$. Then the convenient choice of the normalization point $\lambda_{*}$ is $\lambda_{*}=-\ell \eta$. Indeed, at $\lambda=-\ell \eta$ the series (6.5) terminates at the $k=0$ term; therefore, $\mathrm{Q}^{+}(-\ell \eta)$ is a diagonal operator. Since we did not take care of $\ell$-dependent constant factors, we continue to do so and redefine the $Q$-operator dividing it by the constant $\Gamma^{N}(-4 \ell \eta)$. Note that this 
normalization allows us to define the $Q$-operator for $\ell \in \mathbb{Z}_{+}$as well. We express the result through the function $\Phi_{\ell}$ (A16):

$$
\mathbf{Q}_{L}^{+}(-\ell \eta)=\prod_{j=1}^{N} \Phi_{\ell}^{-1}\left(z_{j}-z_{j+1}\right) \Phi_{\ell}^{-1}\left(z_{j}+z_{j+1}\right)
$$

Thus, by virtue of (5.8), the kernels of the $Q$-operators $\mathbf{Q}^{ \pm}$are

$$
Q^{ \pm}(\lambda ; \vec{\zeta}, \vec{z})=\prod_{i=1}^{N} q^{ \pm}\left(\lambda ; \zeta_{i}, \zeta_{i+1}, z_{i}\right),
$$

where

$$
\begin{aligned}
q^{+}\left(\lambda ; \zeta_{i}, \zeta_{i+1}, z_{i}\right) & =\frac{\Gamma\left(2 \zeta_{i}\right) \Gamma\left(\zeta_{i}-\zeta_{i+1}+2 \lambda\right) \Gamma\left(\zeta_{i}+\zeta_{i+1}+2 \lambda\right)}{\Gamma\left(2 \lambda_{-}\right) \Gamma\left(2 \zeta_{i}+2 \lambda_{+}\right) \Gamma\left(\zeta_{i}-\zeta_{i+1}-2 \ell \eta\right) \Gamma\left(\zeta_{i}+\zeta_{i+1}-2 \ell \eta\right)} \times \\
& \times \sum_{k \geq 0} x_{k}\left(\zeta_{i}, \zeta_{i+1}, \lambda\right) \delta\left(z_{i}-\zeta_{i}-\lambda_{+}+2 k \eta\right)
\end{aligned}
$$

$\left(x_{k}\right.$ is defined in (6.6) $)$, and $q^{-}\left(\lambda, \zeta_{i}, \zeta_{i+1}, z_{i}\right)=q^{+}\left(-\lambda, \zeta_{i+1}, \zeta_{i}, z_{i}\right)$. Representing this in the operator form, according to the rule described in Sec. 3, we obtain:

$$
\begin{aligned}
\mathrm{Q}^{+}(\lambda) & =\frac{1}{\Gamma^{N}(2 \lambda-2 \ell \eta)} \prod_{i=1}^{N} \frac{\Gamma\left(2 z_{i}\right) \Gamma\left(z_{i}-z_{i+1}+2 \lambda\right) \Gamma\left(z_{i}+z_{i+1}+2 \lambda\right)}{\Gamma\left(2 z_{i}+2 \lambda+2 \ell \eta\right) \Gamma\left(z_{i}-z_{i+1}-2 \ell \eta\right) \Gamma\left(z_{i}+z_{i+1}-2 \ell \eta\right)} \times \\
& \times \\
\hline & \prod_{j=1}^{N}{ }_{6} W_{5}\left(-\frac{z_{j}+\lambda}{\eta}-\ell ;-\frac{\lambda}{\eta}-\ell,-\frac{z_{j+1}+z_{j}}{2 \eta}-\ell, \frac{z_{j+1}-z_{j}}{2 \eta}-\ell ; e^{-2 \eta \partial_{z_{j}}} \mid \eta, \tau\right): \times \\
& \times \exp \left((\lambda+\ell \eta) \sum_{m=1}^{N} \partial_{z_{m}}\right) \\
\mathbf{Q}^{-}(\lambda) & =\frac{1}{\Gamma^{N}(-2 \lambda-2 \ell \eta)} \prod_{i=1}^{N} \frac{\Gamma\left(2 z_{i}\right) \Gamma\left(z_{i}-z_{i-1}-2 \lambda\right) \Gamma\left(z_{i}+z_{i-1}-2 \lambda\right)}{\Gamma\left(2 z_{i}-2 \lambda+2 \ell \eta\right) \Gamma\left(z_{i}-z_{i-1}-2 \ell \eta\right) \Gamma\left(z_{i}+z_{i-1}-2 \ell \eta\right)} \times \\
& \times \prod_{j=1}^{N}{ }_{6} W_{5}\left(-\frac{z_{j}-\lambda}{\eta}-\ell ; \frac{\lambda}{\eta}-\ell,-\frac{z_{j}+z_{j-1}}{2 \eta}-\ell, \frac{z_{j}-z_{j-1}}{2 \eta}-\ell ; e^{\left.-2 \eta \partial_{z_{j}} \mid \eta, \tau\right)} \times\right. \\
& \times \exp \left((-\lambda+\ell \eta) \sum_{m=1}^{N} \partial_{z_{m}}\right) \mathbf{C},
\end{aligned}
$$

where $\mathbf{C}$ is the cyclic shift operator,

$$
(\mathbf{C} f)\left(z_{1}, z_{2}, \ldots, z_{N-1}, z_{N}\right)=f\left(z_{2}, z_{3}, \ldots, z_{N}, z_{1}\right),
$$

with the kernel $C(\vec{\zeta}, \vec{z})=\prod_{i=1}^{N} \delta\left(z_{i}-\zeta_{i+1}\right)$. Clearly, $\mathbf{C}$ commutes with the $\mathbf{T}(\lambda)$ and the $\mathbf{Q}^{ \pm}(\lambda)$ 
In general, the series ${ }_{6} W_{5}$ in (77.7), (7.8) is not terminating. However, at $\lambda=\mp \ell \eta \pm m \eta$, $m \in \mathbb{Z}_{+}$, the series ${ }_{6} W_{5}$ in (7.7) (respectively, in (7.8)) terminates at the $m$-th term. Note also that

$$
\mathbf{Q}^{ \pm}( \pm \ell \eta \mp m \eta)=0, \quad m=0,1,2, \ldots,
$$

due to zeros of the scalar common factor. At the points $\pm \ell \eta$ we have:

$$
\mathbf{Q}^{+}(-\ell \eta)=\frac{1}{\Gamma^{N}(-4 \ell \eta)} \mathbf{I}, \quad \mathbf{Q}^{-}(\ell \eta)=\frac{1}{\Gamma^{N}(-4 \ell \eta)} \mathbf{C},
$$

where $\mathbf{I}$ is the identity operator. So, at $\ell \in \frac{1}{2} \mathbb{Z}_{+}$the $Q$-operators have poles at these points.

Let us evaluate the $Q$-operator at another distinguished point. Note that if $\lambda=(\ell+1) \eta$, then $\zeta_{i}$ in the kernel of $\mathbf{Q}^{+}$decouples from $\zeta_{i+1}$, so the operator simplifies:

$$
\mathbf{Q}^{+}((\ell+1) \eta)=\left(\prod_{i=1}^{N} \Phi_{\ell}\left(z_{i}-z_{i+1}\right) \Phi_{\ell}\left(z_{i}+z_{i+1}\right)\right) \prod_{j=1}^{N} \mathbf{w}_{\ell}^{j} .
$$

The operators $\mathbf{w}_{\ell}$ pairwise commute since they act on variables attached to different sites of the chain. From (7.7) one obtains the representation of the operator $\mathbf{w}_{\ell}$ through the elliptic hypergeometric series ${ }_{4} W_{3}$ with an operator argument:

$$
\mathbf{w}_{\ell}=\frac{e^{2 \pi i(2 \ell+1) z} \Gamma(2 z)}{\Gamma(2 z+2(2 \ell+1) \eta)}:{ }_{4} W_{3}\left(-\frac{z}{\eta}-2 \ell-1 ;-2 \ell-1 ; e^{-2 \eta \partial_{z}} \mid \eta, \tau\right): e^{(2 \ell+1) \eta \partial_{z}} .
$$

At $\ell \in \frac{1}{2} \mathbb{Z}_{+}$the series is terminating and gives the operator $\mathbf{w}_{\ell}$ introduced in (3.4). So, at $\lambda=(\ell+1) \eta$ we obtain a distinguished factorized integral of motion

$$
\mathbf{A}=\left(\prod_{i=1}^{N} \Phi_{\ell}\left(z_{i}+z_{i+1}\right) \Phi_{\ell}\left(z_{i}-z_{i+1}\right)\right) \prod_{j=1}^{N} \mathbf{w}_{\ell}
$$

(no normal ordering is required since the $\stackrel{j}{\mathbf{w}}_{\ell}$ commute).

Let us give some remarks on the operator A. It is clear from Sec. 3 that for $\ell \in$ $\frac{1}{2} \mathbb{Z}_{+}$the operator $\mathbf{A}$ annihilates the space $\left(\Theta_{4 \ell}^{+}\right)^{\otimes N}$. At $\ell=0$, this integral of motion is proportional to $\mathbf{T}(0)$ and has especially simple form: $2^{N}\left(\prod_{i=1}^{N} \theta_{1}\left(z_{i}+z_{i+1}\right) \theta_{1}\left(z_{i}-\right.\right.$ $\left.\left.z_{i+1}\right) / \theta_{1}\left(2 z_{i}\right)\right) \prod_{j=1}^{N} \sinh \left(\eta \partial_{z}\right)$. (In the limit to the $X X X$ spin chain it coincides with the integral of motion found by Lipatov [23] in the context of high energy QCD, see also [24].) Presumably, for $\ell \in \frac{1}{2} \mathbb{Z}_{+}$A can be represented as the trace of the product of more general L-operators obtained from (2.1) by the fusion procedure, taken at a particular value of $\lambda$. For brevity, we denote $\varphi_{\ell}\left(z_{i}, z_{i+1}\right)=\Phi_{\ell}\left(z_{i}-z_{i+1}\right) \Phi_{\ell}\left(z_{i}+z_{i+1}\right)$. Together with (3.6), the commutativity of $\mathbf{A}$ and $\mathbf{T}(\lambda)$ implies the intertwining relation

$$
\mathbf{T}^{(\ell)}(\lambda) \prod_{i=1}^{N} \varphi_{\ell}\left(z_{i}, z_{i+1}\right)=\left(\prod_{i=1}^{N} \varphi_{\ell}\left(z_{i}, z_{i+1}\right)\right) \mathbf{T}^{(-\ell-1)}(\lambda),
$$

where the superscript of $\mathbf{T}$ indicates the value of spin. A direct proof of this relation is given in Appendix B. We stress that the intertwining relation (3.6) holds for all the spin operators separately while (7.15) is valid for the transfer matrix only. 
The operators $\mathbf{Q}^{ \pm}(\lambda)$ are two operator solutions of the Baxter $T$ - $Q$ relation $(7.3)$ treated as a difference equation in $\lambda$. Therefore, their Wronskian,

$$
\mathbf{W}(\lambda)=\mathbf{Q}^{+}(\lambda+\eta) \mathbf{Q}^{-}(\lambda)-\mathbf{Q}^{+}(\lambda) \mathbf{Q}^{-}(\lambda+\eta)
$$

obeys the functional equation $\theta_{1}^{N}(2 \lambda-2 \ell \eta) \mathbf{W}(\lambda)=\theta_{1}^{N}(2 \lambda+2 \ell \eta) \mathbf{W}(\lambda-\eta)$. Taking into account (7.10), one can write

$$
\mathbf{W}(\lambda)=\theta_{1}^{N}(2 \lambda+2 \ell \eta \mid 2 \eta)\left(\frac{\Gamma(2 \lambda+2 \ell \eta+2 \eta)}{\Gamma(2 \lambda-2 \ell \eta+2 \eta)}\right)^{N} \mathbf{p}(\lambda) \mathbf{A C}
$$

where $\mathbf{p}(\lambda)$ is a diagonal operator such that $\mathbf{p}(\lambda+1)=\mathbf{p}(\lambda), \mathbf{p}(\lambda+\eta)=e^{2 \pi i N \eta} \mathbf{p}(\lambda)$, and $\mathbf{p}(\ell \eta)=\mathbf{I}$. The operator $\mathbf{A}$ is given in (7.14). (The simplest way to find the operator factors is to calculate the Wronskian at $\lambda=\ell \eta$.) To find $\mathbf{p}(\lambda)$ explicitly, a more careful analysis is required.

\section{Miscellanea}

\section{Twisted boundary conditions}

The twisted boundary condition means inserting a $c$-number matrix inside the trace of the chain of L-operators (2.3). To preserve integrability, this matrix must be a constant solution to the $\mathrm{RLL}=\mathrm{LLR}$ relation. In the elliptic case, there are only three such solutions: the Pauli matrices $\sigma_{\alpha}, \alpha=1,2,3$. Therefore, the definition of the quantum transfer matrix with twisted boundary conditions is as follows:

$$
\mathbf{T}(\lambda)=\operatorname{tr}\left[\stackrel{1}{\mathrm{~L}}(\lambda) \stackrel{2}{\mathrm{~L}}(\lambda) \ldots \stackrel{N}{\mathrm{~L}}(\lambda) \sigma_{\alpha}\right]
$$

For definitness, we consider the case of $\sigma_{1}=\left(\begin{array}{cc}0 & 1 \\ 1 & 0\end{array}\right)$.

Again, we employ the gauge transformation (5.1) with the same matrix (5.2). The whole argument remains the same with the only modification that $\zeta_{N+1} \neq \zeta_{1}$. The $\zeta_{N+1}$ should be adjusted in such a way that the matrix $\sigma_{1}^{\prime}=G\left(\zeta_{N+1}\right) \sigma_{1}\left(G\left(\zeta_{1}\right)\right)^{-1}$ be lowertriangular. Calculating it explicitly, we obtain

$$
\sigma_{1}^{\prime}=\frac{1}{\bar{\theta}_{4}\left(\zeta_{1}\right)}\left(\begin{array}{cc}
\bar{\theta}_{3}\left(\zeta_{N+1}\right) & -2 \theta_{2}\left(\zeta_{1}+\zeta_{N+1}\right) \theta_{2}\left(\zeta_{1}-\zeta_{N+1}\right) \\
1 & -\bar{\theta}_{3}\left(\zeta_{1}\right)
\end{array}\right),
$$

where a simple identity for $\theta$-functions has been used. Therefore, if $\zeta_{N+1}=\zeta_{1}+\frac{1}{2}$, then the right upper element is zero.

With this modification, the definitions of the vacuum vectors (5.4), (5.5) and of the pre$Q$-operators (5.8), (5.9) remain the same. However, equations (5.10), (5.11) get modified:

$$
\begin{aligned}
& \mathbf{T}(\lambda) \mathbf{Q}_{R}^{ \pm}(\lambda)=\theta_{1}^{N}\left(2 \lambda_{-}\right) \mathbf{Q}_{R}^{ \pm}(\lambda+\eta)-\theta_{1}^{N}\left(2 \lambda_{+}\right) \mathbf{Q}_{R}^{ \pm}(\lambda-\eta) \\
& \mathbf{Q}_{L}^{ \pm}(\lambda) \mathbf{T}(\lambda)=\theta_{1}^{N}\left(2 \lambda_{-}\right) \mathbf{Q}_{L}^{ \pm}(\lambda+\eta)-\theta_{1}^{N}\left(2 \lambda_{+}\right) \mathbf{Q}_{L}^{ \pm}(\lambda-\eta)
\end{aligned}
$$


The new constraint on $\zeta_{N+1}$ and $\zeta_{1}$ does not destroy the basic commutation relation (6.1). Indeed, the $g$-factors in (6.16) still cancel in the product over the chain since $g_{\zeta_{1} \zeta_{1}^{\prime}}=g_{\zeta_{N+1} \zeta_{N+1}^{\prime}}$ due to (6.9) and (A7).

The normalization of the $Q$-operators goes through as before. The $T$ - $Q$ relation is

$$
\mathbf{T}(\lambda) \mathbf{Q}(\lambda)=\theta_{1}^{N}(2 \lambda-2 \ell \eta) \mathbf{Q}(\lambda+\eta)-\theta_{1}^{N}(2 \lambda+2 \ell \eta) \mathbf{Q}(\lambda-\eta)
$$

The $Q$-operators are given by the same formulas (7.7) (7.8), where it is implied that $\zeta_{N+1}=\zeta_{1}+\frac{1}{2}$, and the cyclic shift operator $\mathbf{C}$ should be replaced by the operator $\mathbf{C}^{\prime}$ such that $\left(\mathbf{C}^{\prime} f\right)\left(z_{1}, z_{2}, \ldots, z_{N}\right)=f\left(z_{2}, z_{3}, \ldots, z_{N}, z_{1}+\frac{1}{2}\right)$.

The case $N=1$

For $N=1$, there is only one $\mathrm{L}$-operator in the chain (2.3). Trace of the $\mathrm{L}(\lambda)$ is the simplest quantum transfer matrix $\mathbf{T}(\lambda)$. It is proportional to $\mathbf{s}_{0}$ :

$$
\begin{gathered}
\mathbf{T}(\lambda)=\operatorname{tr} \mathrm{L}(\lambda)=\theta_{1}(2 \lambda) \mathbf{s}_{0}, \\
\mathbf{s}_{0}=\frac{\theta_{1}(2 z-2 \ell \eta)}{\theta_{1}(2 z)} e^{\eta \partial_{z}}+\frac{\theta_{1}(2 z+2 \ell \eta)}{\theta_{1}(2 z)} e^{-\eta \partial_{z}} .
\end{gathered}
$$

This is the difference analog of the Lamé operator proposed in [25]. The difference operator given by eq. (8.7) for positive integer values of $\ell$ and arbitrary generic $\eta$ has $2 \ell+1$ stable bands (and $2 \ell$ gaps) in the spectrum.

The construction of the previous sections gives a remarkable family of commuting difference operators in one variable. For $\ell \in \mathbb{Z}_{+}$this family of operators commuting with (8.7) coincides with the one found in [26] by means of a different argument.

The case $N=1$ is exceptional and must be considered separately. Some of the arguments for $N \geq 2$ are not directly applicable to $N=1$ since the vacuum vectors (4.15), (4.17) are to be taken at $\zeta=\xi$ that for $\ell \in \mathbb{Z}_{+}$means the non-generic position of the parameters. Nevertheless, it is a matter of direct verification to prove that the final formulas (7.7), (7.8) remain true, if just to change the normalization making them non-singular for $\ell \in \mathbb{Z}_{+}$:

$$
\begin{aligned}
\mathrm{Q}_{(N=1)}^{ \pm}( \pm \lambda) & =\frac{\Gamma(2 \lambda) \Gamma(2 z) \Gamma(2 z+2 \lambda)}{\Gamma(2 \lambda-2 \ell \eta) \Gamma(2 z-2 \ell \eta) \Gamma(2 z+2 \lambda+2 \ell \eta)} \times \\
& \times:{ }_{6} W_{5}\left(-\frac{z+\lambda}{\eta}-\ell ;-\frac{\lambda}{\eta}-\ell,-\frac{z}{\eta}-\ell,-\ell ; e^{-2 \eta \partial_{z}} \mid \eta, \tau\right): e^{(\lambda+\ell \eta) \partial_{z}} .
\end{aligned}
$$

For $\ell \in \mathbb{Z}_{+}$the series is terminating. In this case the above formula can be rewritten in a more explicit form:

$$
\mathbf{Q}_{(N=1)}^{ \pm}(\lambda)=\sum_{k=0}^{\ell} A_{k}(z, \lambda) e^{(2 k \eta-\ell \eta+\lambda) \partial_{z}}
$$

where

$$
\begin{aligned}
A_{k}(z, \lambda)=(-1)^{k} \frac{[\ell] !}{[2 \ell] !}\left[\begin{array}{l}
\ell \\
k
\end{array}\right] & \prod_{j=0}^{\ell-k-1} \frac{\theta_{1}(2 z+2(\ell-j) \eta) \theta_{1}(2 \lambda+2(\ell-j) \eta)}{\theta_{1}(2 z+2 \lambda+2(k-j) \eta)} \\
& \times \prod_{j=0}^{k-1} \frac{\theta_{1}(2 z-2(\ell-j) \eta) \theta_{1}(2 \lambda-2(\ell-j) \eta)}{\theta_{1}(2 z+2 \lambda+2(k+j-\ell) \eta)} .
\end{aligned}
$$


If $k=0$ or $k=\ell$, then the second (respectively, the first) product is absent.

\section{The $X X Z$-case}

The construction of Secs. $5-7$ admits several different trigonometric degenerations. Here we discuss the one corresponding to the $X X Z$ spin chain with the standard 6 -vertex $R$-matrix. The L-operator is (cf. (2.1))

$$
\mathbf{L}^{(X X Z)}(u)=\frac{1}{2 i}\left(\begin{array}{cc}
e^{2 \pi i u+\mathbf{j}_{0}}-e^{-2 \pi i u-\mathbf{j}_{0}} & \mathbf{j}_{-} \\
\mathbf{j}_{+} & e^{2 \pi i u-\mathbf{j}_{0}}-e^{-2 \pi i u+\mathbf{j}_{0}}
\end{array}\right)
$$

where $u$ is the spectral parameter and $\mathbf{j}_{0}, \mathbf{j}_{ \pm}$are operators acting on functions of a variable $x$ as follows:

$$
\mathbf{j}_{\mathbf{0}}=\gamma \partial_{x}, \quad \mathbf{j}_{ \pm}=e^{ \pm 2 \pi i x}\left(q^{\ell} e^{\mp \gamma \partial_{x}}-q^{-\ell} e^{ \pm \gamma \partial_{x}}\right) .
$$

Here $q=e^{2 \pi i \gamma}$, and these operators obey the commutation relations of the quantum algebra $U_{q}(\operatorname{sl}(2))$ :

$$
\left[\mathbf{j}_{0}, \mathbf{j}_{ \pm}\right]= \pm 2 \pi i \gamma \mathbf{j}_{ \pm}, \quad\left[\mathbf{j}_{+}, \mathbf{j}_{-}\right]=2\left(q-q^{-1}\right) \sinh \mathbf{j}_{0}
$$

The quantum transfer matrix $\mathbf{T}(u)$ is defined by the same formula (2.3).

The limiting transition from the elliptic formulas to the trigonometric ones consists of two steps:

i) The modular transformation $\tau \rightarrow-1 / \tau$ with the change of variables $z_{j}=\tau x_{j}+\frac{1}{4}$,

$$
\lambda=\tau u, \eta=\tau \gamma
$$

ii) Tending $\tau$ to 0 .

Then the $\mathrm{L}$-operator defined as $\mathrm{L}^{(X X Z)}(u)=\lim _{\tau \rightarrow i 0} \sqrt{\frac{i \tau}{4}} e^{\frac{i \pi}{4 \tau}} \mathrm{L}(\lambda)$ with the understanding that $\tau$ in the $\mathrm{L}(\lambda)$ (2.1) is replaced by $-1 / \tau$, and $u=\lambda / \tau$ is kept fixed coincides with (8.11). The same limiting transition in (7.7) yields the trigonometric $Q$-operator.

Skipping the details (for the modular transformation of the $\theta$-functions and the elliptic gamma-function see eqs. (A4), (A11), respectively), we present the final result. It is written in terms of the following special functions. The basic hypergeometric function ${ }_{2} \phi_{1}$ [27] is defined as

$$
{ }_{2} \phi_{1}\left[\begin{array}{c}
a, b \\
c
\end{array} ; q, x\right]=\sum_{k=0}^{\infty} \frac{(a ; q)_{k}(b ; q)_{k}}{(q ; q)_{k}(c ; q)_{k}} x^{k},
$$

where $(a ; q)_{k}=(1-a)(1-a q)\left(1-a q^{2}\right) \ldots\left(1-a q^{k-1}\right)$. Up to simple redefinitions of parameters, this series gives the little $q$-Jacobi function (when the series is terminating, it is the little $q$-Jacobi polynomial). The $q$-gamma-function is

$$
\Gamma_{q}(x)=\frac{(q ; q)_{\infty}}{\left(q^{x} ; q\right)_{\infty}}(1-q)^{1-x}
$$


where, for $|q|<1,(a ; q)_{\infty}=\lim _{k \rightarrow \infty}(a ; q)_{k}$. The $Q$-operator is given by

$$
\begin{aligned}
& \mathbf{Q}^{+}(u)=e^{-2 \pi i N \ell u} \prod_{i=1}^{N} \frac{\Gamma_{q^{2}}\left(\frac{x_{i}-x_{i+1}+2 u}{2 \gamma}\right)}{\Gamma_{q^{2}}\left(\frac{u}{\gamma}-\ell\right) \Gamma_{q^{2}}\left(\frac{x_{i}-x_{i+1}}{2 \gamma}-\ell\right)} \times \\
& \times: \prod_{j=1}^{N}{ }_{2} \phi_{1}\left[\begin{array}{c}
q^{-2 \ell} e^{-4 \pi i u}, q^{-2 \ell} e^{2 \pi i\left(x_{j+1}-x_{j}\right)} \\
q^{2} e^{2 \pi i\left(x_{j+1}-x_{j}-2 u\right)}
\end{array} ; q^{2}, q^{2(\ell+1)} e^{-2 \gamma \partial_{x_{j}}}\right]: \times \\
& \times \exp \left((u+\ell \gamma) \sum_{m=1}^{N} \partial_{x_{m}}\right) .
\end{aligned}
$$

As in (7.8), the formula for $\mathrm{Q}^{-}(u)$ differs from (8.16) by the changes $u \rightarrow-u, x_{i} \leftrightarrow x_{i+1}$, and the additional cyclic shift $\mathbf{C}$. The both operators $\mathbf{Q}^{ \pm}(u)$ obey the $T$ - $Q$ relation:

$$
\mathbf{T}(u) \mathbf{Q}(u)=\sin ^{N} 2 \pi(u-\ell \gamma) \mathbf{Q}(u+\gamma)+\sin ^{N} 2 \pi(u+\ell \gamma) \mathbf{Q}(u-\gamma)
$$

It is worth noting that the limit from $(7.7)$ gives an additional operator factor which is omitted in (8.16) for it belongs to the center of the algebra generated by the shifts $e^{2 \gamma \partial_{x_{j}}}$ and the operator $\sum_{i=1}^{N} \partial_{x_{i}}$. This factor has the form

$$
\prod_{i=1}^{N} \frac{\Gamma_{\tilde{q}^{2}}(-2 u+2 \ell \gamma+1) \Gamma_{\tilde{q}^{2}}\left(x_{i+1}-x_{i}+2 \ell \gamma+1\right)}{\Gamma_{\tilde{q}^{2}}\left(x_{i+1}-x_{i}-2 u+1\right)},
$$

where $\tilde{q}=\exp \left(-\frac{\pi i}{2 \gamma}\right)$ is the modular partner of the $q=\exp (2 \pi i \gamma)$ (cf. 22]). It seems so that the non-degenerate Sklyanin algebra knows about both halves of the modular double.

\section{Concluding remarks}

In this work, using Baxter's method of vacuum vectors, we have constructed a family of commuting difference operators in many variables. This family provides the $Q$-operator for the quantum integrable spin chain of $X Y Z$-type in the functional realization of the algebra of observables.

The form of the obtained $Q$-operators suggests a simple derivation of local integrals of motion for the $X Y Z$ chain in infinite-dimensional representations. For instance, the natural candidates for local Hamiltonians are $\left(\mathbf{Q}^{ \pm}(\mp \ell \eta)\right)^{-1} d /\left.d \lambda\right|_{\lambda=\mp \ell \eta} \mathbf{Q}^{ \pm}(\lambda)$. Their explicit form is, however, not very illuminating. The hermiticity properties of these operators are to be further investigated. We leave this for a future work.

Another important point remaining out of the scope of this paper is the limit to the $X X X$-case $(\gamma \rightarrow 0$ in (8.11) $)$. In this limit one should be able to link our results with the ones of the papers [6, 0, 11], where the $Q$-operator for the $X X X$ spin chain and allied models with rational $R$-matrix was realized as an integral operator. However, it is hard to compare them directly since the limit is singular and, therefore, very delicate. Moreover, as it should be clear from the above, the rational limit is certainly not unique. Different rational limits of the trigonometric $\mathrm{L}$-operator lead to $\mathrm{L}$-operators for the $X X X$ spin chains, where the $s l(2)$-algebra is realized in different ways. A detailed study of rational limits seems to be interesting since in this case, closely related to the representation theory 
of $S L(2, \mathbb{R})$ or $S U(1,1)$, the result must admit a meaningful translation into the language of representation theory and harmonic analysis. This may give a hint of how an elliptic extension of the latter might look like.

The construction of the pre- $Q$-operators, $\mathbf{Q}_{L}(\lambda)$ and $\mathbf{Q}_{R}(\lambda)$, goes through also for inhomogeneous chains. The problem, however, is the normalization: in general there is no point $\lambda_{*}$ such that $\mathbf{Q}_{L}\left(\lambda_{*}\right)$ or $\mathbf{Q}_{R}\left(\lambda_{*}\right)$ could be explicitly inverted. Generally speaking, the kernel of the $Q$-operator can not be represented as a product of local factors, so local integrals of motion for inhomogeneous chains probably do not exist. In other respects, the pair of pre- $Q$-operators is a good substitute for the $Q$-operator. Their kernels are factorized though the operators do not commute. Presumably, they still obey the exchange commutation relations (6.1). Their proof given in Sec. 6 should basically go through in the inhomogeneous case, too, since the key element of the proof is local in nature.

At last, we note that the technique developed in the present paper might help to construct the fundamental $R$-matrix in infinite-dimensional representations of the Sklyanin algebra.

\section{Acknowledgments}

I am grateful to E.Antonov, S.Kharchev, S.Khoroshkin, I.Krichever, A.Levin, and T.Takebe for useful discussions, and to V.Spiridonov for bringing the paper [17] to my attention. This work was supported in part by RFBR grant 98-01-00344.

\section{Appendix A}

\section{Theta-functions}

We use the following definition of the Jacobi $\theta$-functions:

$$
\begin{aligned}
& \theta_{1}(z \mid \tau)=-\sum_{k \in \mathbb{Z}} \exp \left(\pi i \tau\left(k+\frac{1}{2}\right)^{2}+2 \pi i\left(z+\frac{1}{2}\right)\left(k+\frac{1}{2}\right)\right) \\
& \theta_{2}(z \mid \tau)=\sum_{k \in \mathbb{Z}} \exp \left(\pi i \tau\left(k+\frac{1}{2}\right)^{2}+2 \pi i z\left(k+\frac{1}{2}\right)\right) \\
& \theta_{3}(z \mid \tau)=\sum_{k \in \mathbb{Z}} \exp \left(\pi i \tau k^{2}+2 \pi i z k\right) \\
& \theta_{4}(z \mid \tau)=\sum_{k \in \mathbb{Z}} \exp \left(\pi i \tau k^{2}+2 \pi i\left(z+\frac{1}{2}\right) k\right)
\end{aligned}
$$

They also can be represented as infinite products. The infinite product representation for the $\theta_{1}(z \mid \tau)$ reads:

$$
\theta_{1}(z \mid \tau)=i \exp \left(\frac{i \pi \tau}{4}-i \pi z\right) \prod_{k=1}^{\infty}\left(1-e^{2 \pi i k \tau}\right)\left(1-e^{2 \pi i((k-1) \tau+z)}\right)\left(1-e^{2 \pi i(k \tau-z)}\right)
$$


Throughout the paper we write $\theta_{a}(x \mid \tau)=\theta_{a}(x)$. The transformation properties for shifts by the periods are:

$$
\theta_{a}(x \pm 1)=(-1)^{\delta_{a, 1}+\delta_{a, 2}} \theta_{a}(x), \quad \theta_{a}(x \pm \tau)=(-1)^{\delta_{a, 1}+\delta_{a, 4}} e^{-\pi i \tau \mp 2 \pi i x} \theta_{a}(x) .
$$

Under the modular transformation $\tau \rightarrow-1 / \tau$ the $\theta$-functions behave as follows:

$$
\begin{aligned}
& \theta_{1}(z \mid \tau)=i \sqrt{i / \tau} e^{-\pi i z^{2} / \tau} \theta_{1}(z / \tau \mid-1 / \tau) \\
& \theta_{2}(z \mid \tau)=\sqrt{i / \tau} e^{-\pi i z^{2} / \tau} \theta_{4}(z / \tau \mid-1 / \tau) \\
& \theta_{3}(z \mid \tau)=\sqrt{i / \tau} e^{-\pi i z^{2} / \tau} \theta_{3}(z / \tau \mid-1 / \tau) \\
& \theta_{4}(z \mid \tau)=\sqrt{i / \tau} e^{-\pi i z^{2} / \tau} \theta_{2}(z / \tau \mid-1 / \tau)
\end{aligned}
$$

By $\Theta_{n}$ we denote the space of $\theta$-functions of order $n$, i.e., entire functions $F(x), x \in \mathbb{C}$, such that

$$
F(x+1)=F(x), \quad F(x+\tau)=(-1)^{n} e^{-\pi i n \tau-2 \pi i n x} F(x) .
$$

It is easy to see that $\operatorname{dim} \Theta_{n}=n$. Let $F(x) \in \Theta_{n}$, then $F(x)$ has a multiplicative representation of the form $F(x)=c \prod_{i=1}^{n} \theta_{1}\left(x-x_{i}\right), \sum_{i=1}^{n} x_{i}=0$, where $c$ is a constant. Imposing, in addition to (A5), the condition $F(-x)=F(x)$, we define the space $\Theta_{n}^{+} \subset \Theta_{n}$ of even $\theta$-functions of order $n$, which plays the important role in representations of the Sklyanin algebra. If $n$ is an even number, then $\operatorname{dim} \Theta_{n}^{+}=\frac{1}{2} n+1$.

\section{Elliptic gamma-function}

Here we collect the main formulas on the elliptic gamma-function [18, 19]. We use the (slightly modified) notation of [19]. The elliptic gamma-function is defined by the doubleinfinite product

$$
\Gamma\left(z \mid \tau, \tau^{\prime}\right)=\prod_{k, k^{\prime}=0}^{\infty} \frac{1-e^{2 \pi i\left((k+1) \tau+\left(k^{\prime}+1\right) \tau^{\prime}-z\right)}}{1-e^{2 \pi i\left(k \tau+k^{\prime} \tau^{\prime}+z\right)}}
$$

A sufficient condition for the product to be convergent is $\operatorname{Im} \tau>0, \operatorname{Im} \tau^{\prime}>0$. We need the following properties of the elliptic gamma-function:

$$
\begin{gathered}
\Gamma\left(z+1 \mid \tau, \tau^{\prime}\right)=\Gamma\left(z \mid \tau, \tau^{\prime}\right) \\
\Gamma\left(z+\tau \mid \tau, \tau^{\prime}\right)=-i e^{-\frac{\pi i \tau^{\prime}}{6}} \eta_{D}^{-1}\left(\tau^{\prime}\right) e^{\pi i z} \theta_{1}\left(z \mid \tau^{\prime}\right) \Gamma\left(z \mid \tau, \tau^{\prime}\right) \\
\Gamma\left(z+\tau^{\prime} \mid \tau, \tau^{\prime}\right)=-i e^{-\frac{\pi i \tau}{6}} \eta_{D}^{-1}(\tau) e^{\pi i z} \theta_{1}(z \mid \tau) \Gamma\left(z \mid \tau, \tau^{\prime}\right)
\end{gathered}
$$

where

$$
\eta_{D}(\tau)=e^{\frac{\pi i \tau}{12}} \prod_{k=1}^{\infty}\left(1-e^{2 \pi i k \tau}\right)
$$

is the Dedekind function. Another useful property is

$$
\Gamma\left(z \mid \tau, \tau^{\prime}\right) \Gamma\left(\tau^{\prime}-z \mid \tau, \tau^{\prime}\right)=\frac{i e^{\pi i \tau^{\prime} / 6} \eta_{D}\left(\tau^{\prime}\right)}{e^{\pi i z} \theta_{1}\left(z \mid \tau^{\prime}\right)} .
$$


Under the modular transformation $\tau \rightarrow-1 / \tau$ the elliptic gamma-function behaves as follows [19]:

$$
\Gamma\left(z \mid \tau, \tau^{\prime}\right)=e^{i \pi P(z)} \frac{\Gamma\left(z / \tau \mid-1 / \tau, \tau^{\prime} / \tau\right)}{\Gamma\left((z-\tau) / \tau^{\prime} \mid-\tau / \tau^{\prime},-1 / \tau^{\prime}\right)},
$$

where

$$
\begin{aligned}
P(z) & =-\frac{1}{3 \tau \tau^{\prime}} z^{3}+\frac{\tau+\tau^{\prime}-1}{2 \tau \tau^{\prime}} z^{2}-\frac{\tau^{2}+\tau^{\prime 2}+3 \tau \tau^{\prime}-3 \tau-3 \tau^{\prime}+1}{6 \tau \tau^{\prime}} z- \\
& -\frac{\left(\tau+\tau^{\prime}-1\right)\left(\tau+\tau^{\prime}-\tau \tau^{\prime}\right)}{12 \tau \tau^{\prime}} .
\end{aligned}
$$

Let us list the most frequently used formulas for $\Gamma(z) \equiv \Gamma(z \mid \tau, 2 \eta)$. Using (A9) several times, we obtain:

$$
\begin{gathered}
\frac{\Gamma(x+2 k \eta)}{\Gamma(x)}=e^{\pi i \eta k^{2}} R^{-k} e^{\pi i k x} \prod_{j=0}^{k-1} \theta_{1}(x+2 j \eta), \\
\frac{\Gamma(x-2 k \eta)}{\Gamma(x)}=(-1)^{k} e^{\pi i \eta k^{2}} R^{k} e^{-\pi i k x} \prod_{j=0}^{k-1}\left(\theta_{1}(-x+2 \eta+2 j \eta)\right)^{-1},
\end{gathered}
$$

where $R=i e^{\pi i(\eta+\tau / 6)} \eta_{D}(\tau)$.

As is seen from ( $\overline{A 6})$, the function $\Gamma(z \mid \tau, 2 \eta)$ has zeros at the points $z=2(k+1) \eta+$ $(m+1) \tau+n$, and simple poles at the points $z=-2 k \eta-m \tau+n$, where $k, m$ run over non-negative integers and $n$ over all integers. The residues of the elliptic gamma-function at the poles at $z=-2 k \eta, k=0,1,2, \ldots$ are:

$$
\text { res }\left.\right|_{z=-2 k \eta} \Gamma(z)=(-1)^{k} e^{\pi i \eta k^{2}} R^{k} r_{0} \prod_{j=1}^{k}\left(\theta_{1}(2 j \eta)\right)^{-1}
$$

where

$$
r_{0}=\left.\operatorname{res}\right|_{z=0} \Gamma(z)=-\frac{e^{\pi i(\tau+2 \eta) / 12}}{2 \pi i \eta_{D}(\tau) \eta_{D}(2 \eta)} .
$$

The function $\Phi_{\ell}(z)$ (4.14) can be extended to arbitrary complex values of $\ell$ as follows:

$$
\Phi_{\ell}(z)=e^{-\pi i \eta(2 \ell+1)} R^{2 \ell+1} e^{-\pi i(2 \ell+1) z} \frac{\Gamma(z+2(\ell+1) \eta)}{\Gamma(z-2 \ell \eta)} .
$$

\section{Elliptic hypergeometric series}

Here we follow [17]. We define the elliptic Pochhammer symbol (the shifted elliptic factorial) by

$$
[x]_{k} \equiv[x][x+1] \ldots[x+k-1],
$$

where $[x]=\theta_{1}(2 x \eta)$ (cf. (3.5)). By definition, the elliptic hypergeometric series is

$$
{ }_{r+1} W_{r}\left(\alpha_{1} ; \alpha_{4}, \alpha_{5}, \ldots, \alpha_{r+1} ; z \mid \eta, \tau\right)=\sum_{k=0}^{\infty} z^{k} \frac{\left[\alpha_{1}+2 k\right]\left[\alpha_{1}\right]_{k}}{\left[\alpha_{1}\right][k] !} \prod_{m=1}^{r-2} \frac{\left[\alpha_{m+3}\right]_{k}}{\left[\alpha_{1}-\alpha_{m+3}+1\right]_{k}} .
$$


This is an elliptic analog of the very-well-poised basic hypergeometric series [27]. The series is said to be balanced if $z=1$ and

$$
r-5+(r-3) \alpha_{1}=2 \sum_{m=1}^{r-2} \alpha_{m+3} .
$$

For a series $\sum_{k \geq 0} c_{k}$ of the form (A18), the balancing condition (A19) means that the ratio $c_{k+1} / c_{k}$ of the coefficients is an elliptic function of $k$. For balanced series (A18), we drop the argument $z=1$ and the parameters $\eta, \tau$ writing it simply as ${ }_{r+1} W_{r}\left(\alpha_{1} ; \alpha_{4}, \ldots, \alpha_{r+1}\right)$. For instance,

$$
{ }_{10} W_{9}\left(\alpha_{1} ; \alpha_{4}, \alpha_{5}, \ldots, \alpha_{10}\right)=\sum_{k=0}^{\infty} \frac{\left[\alpha_{1}+2 k\right]\left[\alpha_{1}\right]_{k}}{\left[\alpha_{1}\right][k] !} \prod_{m=1}^{7} \frac{\left[\alpha_{m+3}\right]_{k}}{\left[\alpha_{1}-\alpha_{m+3}+1\right]_{k}} .
$$

The series is called terminating if at least one of the parameters $\alpha_{4}, \ldots, \alpha_{r+1}$ is equal to a negative integer number. In this case there is no problem of convergence. If, say $\alpha_{r+1}=-n$, then the series terminates at $k=n$. The terminating balanced hypergeometric series were shown [17] to possess nice modular properties. That is why they were called modular hypergeometric series.

The modular hypergeometric series obey a number of spectacular identities. One of them is the elliptic analog of the Bailey transformation for the ${ }_{10} W_{9}$ [27:

$$
\begin{aligned}
& { }_{10} W_{9}\left(\alpha_{1} ; \alpha_{4}, \ldots, \alpha_{10}\right) \\
= & { }_{10} W_{9}\left(\beta_{1} ; \beta_{4}, \ldots, \beta_{10}\right) \frac{\left[\alpha_{1}+1\right]_{n}\left[\beta_{1}-\beta_{7}+1\right]_{n}\left[\beta_{1}-\beta_{8}+1\right]_{n}\left[\alpha_{1}-\alpha_{7}-\alpha_{8}+1\right]_{n}}{\left[\beta_{1}+1\right]_{n}\left[\alpha_{1}-\alpha_{7}+1\right]_{n}\left[\alpha_{1}-\alpha_{8}+1\right]_{n}\left[\beta_{1}-\beta_{7}-\beta_{8}+1\right]_{n}},
\end{aligned}
$$

where $\alpha_{j}$ obey the balancing condition $2+3 \alpha_{1}=\sum_{m=1}^{7} \alpha_{m+3}, \alpha_{10}=-n$, and the parameters $\beta_{j}$ are as follows:

$$
\begin{array}{ll}
\beta_{1}=2 \alpha_{1}+1-\alpha_{4}-\alpha_{5}-\alpha_{6}, & \\
\beta_{i}=\beta_{1}-\alpha_{1}+\alpha_{i}, & i=4,5,6, \\
\beta_{i}=\alpha_{i}, & i=7, \ldots, 10 .
\end{array}
$$

This identity was proved in 17].

\section{Appendix B}

Here we present the proofs of the intertwining relations (3.6) and the commutation relation (7.15).

For general complex values of $\ell$ the operator $\mathbf{w}_{\ell}$ is given by the series (7.13):

$$
\mathbf{w}_{\ell}=\frac{e^{2 \pi i(2 \ell+1) z} \Gamma(2 z)}{\Gamma(2 z+2(2 \ell+1) \eta)} \bullet_{4} W_{3}\left(-\frac{z}{\eta}-2 \ell-1 ;-2 \ell-1 ; e^{-2 \eta \partial_{z}} \mid \eta, \tau\right): e^{(2 \ell+1) \eta \partial_{z}}
$$

The proof of (3.6) is a straightforward computation. Substituting (2.2), (B1) into (3.6) and comparing the coefficients of $e^{2(\ell+1-k) \eta \partial_{z}}, k>0$, we get, after cancelling out common 
factors:

$$
\begin{aligned}
& \theta_{1}(2(\ell+k+1) \eta) \theta_{a+1}(2 z+2(\ell+1) \eta) \theta_{1}(2 z+4 k \eta) \theta_{1}(2 z-2(\ell-k+1) \eta) \\
+ & \theta_{1}(2(\ell-k+1) \eta) \theta_{a+1}(-2 z+2(\ell+1) \eta) \theta_{1}(2 z+4 k \eta) \theta_{1}(2 z+2(\ell+k+1) \eta) \\
= & \theta_{1}(2(\ell+k+1) \eta) \theta_{a+1}(2 z+2(2 k-\ell-1) \eta) \theta_{1}(2 z) \theta_{1}(2 z+2(\ell+k+1) \eta) \\
+ & \theta_{1}(2(\ell-k+1) \eta) \theta_{a+1}(-2 z-2(2 k+\ell+1) \eta) \theta_{1}(2 z) \theta_{1}(2 z-2(\ell-k+1) \eta) .
\end{aligned}
$$

All the four terms, as functions of $2 z$, have the same monodromy properties. They are $\theta$-functions of 3 -d order. The linear space of such functions is 3 -dimensional, so it is enough to verify the equality at 3 points, say $z=0, z=-2 k \eta$, and $z=(\ell-k+1) \eta$. The coefficients of the highest shift $e^{2(\ell+1) \eta \partial_{z}}$ in both sides of (3.6) are equal, too, so the intertwining relation is proved.

To prove (7.15), we use the representation (3.2). For any two adjacent sites of the chain (say 1 and 2) we define the $c$-number matrix $\stackrel{12}{M}=\stackrel{12}{M}(\lambda, \ell)=V^{-1}\left(\lambda_{-}, z_{1}\right) V\left(\lambda_{+}, z_{2}\right)$ explicitly given by

$$
\stackrel{12}{M}(\lambda, \ell)=\frac{1}{\theta_{1}\left(2 z_{2}\right)}\left(\begin{array}{cc}
\theta_{1}\left(z_{1}+z_{2}-2 \ell \eta\right) \theta_{1}\left(z_{1}-z_{2}+2 \lambda\right) & -\theta_{1}\left(z_{1}-z_{2}-2 \ell \eta\right) \theta_{1}\left(z_{1}+z_{2}+2 \lambda\right) \\
\theta_{1}\left(z_{1}-z_{2}+2 \ell \eta\right) \theta_{1}\left(z_{1}+z_{2}-2 \lambda\right) & -\theta_{1}\left(z_{1}+z_{2}+2 \ell \eta\right) \theta_{1}\left(z_{1}-z_{2}-2 \lambda\right)
\end{array}\right)
$$

Using the cyclic property of the trace, one can rewrite (2.3) as follows:

$$
\mathbf{T}(\lambda)=\operatorname{tr}\left[\stackrel{12}{M}\left(\begin{array}{cc}
e^{\eta \partial_{2}} & 0 \\
0 & e^{-\eta \partial_{2}}
\end{array}\right) \stackrel{23}{M}\left(\begin{array}{cc}
e^{\eta \partial_{3}} & 0 \\
0 & e^{-\eta \partial_{3}}
\end{array}\right) \ldots \stackrel{N 1}{M}\left(\begin{array}{cc}
e^{\eta \partial_{1}} & 0 \\
0 & e^{-\eta \partial_{1}}
\end{array}\right)\right]:
$$

where $\partial_{i}=\partial_{z_{i}}$. Computing the trace, we obtain:

$$
\mathbf{T}(\lambda)=\sum_{i_{1}, \ldots, i_{N}=1,2} \stackrel{12}{M}_{i_{1} i_{2}}^{2} \stackrel{23}{M}_{i_{2} i_{3}} \ldots \stackrel{N 1}{M} i_{i_{N} i_{1}} e^{\left(3-2 i_{1}\right) \eta \partial_{1}} e^{\left(3-2 i_{2}\right) \eta \partial_{2}} \ldots e^{\left(3-2 i_{N}\right) \eta \partial_{N}} .
$$

Recall that $\varphi_{\ell}\left(z_{i}, z_{i+1}\right)=\Phi_{\ell}\left(z_{i}-z_{i+1}\right) \Phi_{\ell}\left(z_{i}+z_{i+1}\right)$. The commutation relation (7.15),

$$
\mathbf{T}^{(\ell)}(\lambda) \prod_{i=1}^{N} \varphi_{\ell}\left(z_{i}, z_{i+1}\right)=\left(\prod_{i=1}^{N} \varphi_{\ell}\left(z_{i}, z_{i+1}\right)\right) \mathbf{T}^{(-\ell-1)}(\lambda),
$$

follows from (B2) and the local commutation rule

$$
\begin{aligned}
& e^{\left(3-2 i_{1}\right) \eta \partial_{1}+\left(3-2 i_{2}\right) \eta \partial_{2}} \varphi_{\ell}\left(z_{1}, z_{2}\right) \\
= & \frac{\theta_{1}\left(z_{1}+(-1)^{i_{1}+i_{2}} z_{2}-2(-1)^{i_{1}}(\ell+1) \eta\right)}{\theta_{1}\left(z_{1}+(-1)^{i_{1}+i_{2}} z_{2}+2(-1)^{i_{1} \ell}\right)} \varphi_{\ell}\left(z_{1}, z_{2}\right) e^{\left(3-2 i_{1}\right) \eta \partial_{1}+\left(3-2 i_{2}\right) \eta \partial_{2}}, \quad i_{1}, i_{2}=1,2,
\end{aligned}
$$

which is easy to verify. Writing out the entries of the matrix $M$ in the form

$$
\stackrel{12}{M}{ }_{i k}=\theta_{1}\left(z_{1}+(-1)^{i+k} z_{2}+2(-1)^{i} \ell \eta\right) C_{i k}, \quad i, k=1,2,
$$

(the second factor stands for something that does not depend on $\ell$ ), and subsequently moving the factors $\varphi_{\ell}\left(z_{i}, z_{i+1}\right)$ from the right to the left through each term of (B2), we arrive at (7.15). 


\section{References}

[1] R.Baxter, Partition function of the eight-vertex lattice model, Ann. Phys. 70 (1972) 193-228; R.Baxter, Eight-vertex model in lattice statistics and one-dimensional anisotropic Heisenberg chain. I, II, III, Ann. Phys. 76 (1973) 1-24, 25-47, 48-71

[2] L.D.Faddeev and L.A.Takhtadzhan, The quantum method of the inverse problem and the Heisenberg XYZ model (English translation), Russian Math. Surveys 34:5 (1979) $11-68$

[3] I.G.Korepanov, Vacuum curves of $\mathcal{L}$-operators connected with the six-vertex model and construction of $\mathcal{R}$-operators, Chelyabinsk Polytechn. Inst., Deposited in the VINITI 02.04.86, N 2271-B86; Algebra i Analiz 6:2 (1994) 176-194; V.V.Bazhanov and Yu.G.Stroganov, Chiral Potts model as a descendant of the six-vertex model, J. Stat. Phys. 59 (1990) 799-817

[4] M.Batchelor, R.Baxter, M.O'Rourke and C.Yung, Exact solution and interfacial tension of the six-vertex model with anti-periodic boundary conditions, J. Phys. A 28 (1995) 2759-2770; M.Batchelor and C.Yung, Exact solution for the spin-s XXZ quantum chain with non-diagonal twists, Nucl. Phys. B446 (1995) 461-484

[5] V.Pasquier and M.Gaudin, The periodic Toda chain and a matrix generalization of the Bessel function recursion relations, J. Phys. A 28 (1992) 5243-5252

[6] S.Derkachov, Baxter's Q-operator for the homogeneous XXX spin chain, solvint/9902015

[7] G.Pronko, On the Baxter's Q-operator for the $X X X$ spin chain, hep-th/9908179

[8] V.Bazhanov, S.Lukyanov and A.Zamolodchikov, Integrable structure of conformal field theory II. Q-operator and DDV equation, Commun. Math. Phys. 190 (1997) 247-278; Integrable structure of conformal field theory III. The Yang-Baxter relation, Commun. Math. Phys. 200 (1999) 297-324

[9] A.Antonov and B.Feigin, Quantum group representations and Baxter equation, Phys. Lett. B392 (1997) 115-122

[10] V.Kuznetsov and E.Sklyanin, On Bäcklund transformations for many-body systems, J. Phys. A 31 (1998) 2241-2251

[11] V.Kuznetsov, M.Salerno and E.Sklyanin, Quantum Bäcklund transformation for the integrable BST model, solv-int/9908002

[12] T.Takebe, Generalized Bethe ansatz with the general spin representation of the Sklyanin algebra, J. Phys. A 25 (1992) 1071-1083; Bethe ansatz for higher spin eight vertex models, J. Phys. A 28 (1995) 6675-6706

[13] V.Bazhanov and N.Reshetikhin, Critical RSOS models and conformal field theory, Int. J. Mod. Phys. A4 (1989) 115-142

[14] I.Krichever, O.Lipan, P.Wiegmann and A.Zabrodin, Quantum integrable models and discrete classical Hirota equations, Commun. Math. Phys. 188 (1997) 267-304 
[15] E.K.Sklyanin, On some algebraic structures related to the Yang-Baxter equation, Funk. Anal. i ego Pril. 16:4 (1982) 27-34

[16] E.K.Sklyanin, On some algebraic structures related to the Yang-Baxter equation. Representations of the quantum algebra, Funk. Anal i ego Pril. 17:4 (1983) 34-48

[17] I.Frenkel and V.Turaev, Elliptic solutions of the Yang-Baxter equation and modular hypergeometric functions, in: The Arnold-Gelfand Mathematical Seminars, Birkhäuser, Boston, MA, 1997, 171-204

[18] S.N.M.Ruijsenaars, First order analytic difference equations and integrable quantum systems, J. Math. Phys. 38 (1997) 1069-1146

[19] G.Felder and A.Varchenko, The elliptic gamma function and $S L(3, \mathbb{Z}) \times \mathbb{Z}^{3}$, math.QA/9907061

[20] A.Zabrodin, On the spectral curve of the difference Lamé operator, Int. Math. Research Notices No.11 (1999) 589-614

[21] I.Krichever, Baxter's equations and algebraic geometry, Funk. Anal. i ego Pril. 15:2 (1981) 22-35

[22] L.D.Faddeev, Modular double of quantum group, math.QA/9912078

[23] L.N.Lipatov, High energy asymptotics of multi-colour QCD and exactly solvable lattice models, JETP Lett. 59 (1994) 571-574

[24] L.Faddeev and G.Korchemsky, High energy QCD as a completely integrable model, Phys. Lett. B342 (1995) 311-322

[25] I.Krichever and A.Zabrodin, Spin generalization of the Ruijsenaars-Schneider model, non-abelian 2D Toda chain and representations of Sklyanin algebra, Usp. Mat. Nauk, 50:6 (1995) 3-56, hep-th/9505039

[26] G.Felder and A.Varchenko, Algebraic integrability of the two-body Ruijsenaars operator, Funk. Anal. i ego Pril. 32 (1998) 8-25, q-alg/9610024

[27] G.Gasper and M.Rahman, Basic hypergeometric series, Encyclopedia of Mathematics and its Applications, Volume 35, Cambridge University Press, 1990 